\title{
On the relevance of matter and glue dynamics for baryon number fluctuations
}

\author{
Wei-jie $\mathrm{Fu}^{1}$ and Jan M. Pawlowski ${ }^{1,2}$ \\ ${ }^{1}$ Institut für Theoretische Physik, Universität Heidelberg, Philosophenweg 16, 69120 Heidelberg, Germany \\ ${ }^{2}$ ExtreMe Matter Institute EMMI, GSI, Planckstr. 1, 64291 Darmstadt, Germany
}

\begin{abstract}
We investigate the impact of the matter and glue dynamics on baryon number fluctuations and the kurtosis of baryon number distribution. This is done within the framework of QCD-improved low energy effective models. In particular we include the momentum scale dependence of the quarkmeson scattering and the non-trivial dispersions of both, quarks and mesons. On the gluonic side we take into account the backreaction of the matter sector on the glue dynamics. It is shown that the above fluctuations lead to a more rapid change of the baryon number fluctuations as well as the kurtosis of with the chiral crossover.

We also study the signatures of quark confinement in low energy QCD. It is shown that contrary to the common picture the effective thermal distribution in the presence of confining glue backgrounds does not tend towards the colourless baryonic one. Instead, the dominance of colourless hadronic states is obtained in a subtle interplay of quark and glue contributions to the canonical potential.
\end{abstract}

PACS numbers: 11.30.Rd, 11.10.Wx, 05.10.Cc, 12.38.Mh

\section{INTRODUCTION}

Studies of the QCD phase structure and the QCD thermodynamics have attracted lots of attentions in recent years. QCD matter composed of deconfined, coloured quarks and gluons, i.e. the Quark-Gluon Plasma, produced before the quark-hadron transition at $\sim 10 \mu \mathrm{s}$ in the evolution of early Universe 1, is believed to be recreated in heavy-ion collisions at Relativistic Heavy-Ion Colliders such as (RHIC) [2, 3] and LHC [4].

The chiral and confinement-deconfinement crossovers for QCD with physical quark masses at vanishing density may turn into first order transitions at high density. One of the key challenges concerning the phase structure of QCD is to get hold of the existence and location of the corresponding critical end point (CEP), 5. The Beam Energy Scan (BES) program at RHIC is directly aimed at this task, where the beam energy or collision centrality dependence of the fluctuations of conserved charges, such as moments of net-proton multiplicity distributions, is employed to locate the QCD critical point.

In turn, the experimental programme should be accompanied by reliable theoretical predictions for the above observables and their relation to the CEP are highly demanded. A particularly promising direction is the computation of the fluctuation of conserved charges at vanishing and finite density, see e.g. 6 6]. Moreover, it has been suggested that the values of these observables at small density already give access to the existence and location of the CEP, see e.g. [10.

In the present work we investigate the QCD phase structure and thermodynamics as well as baryon number fluctuations within the quark-meson (QM) model and the QCD-enhanced Polyakov-quark-meson (PQM) model. The thermal and quantum dynamics of the models is accessed with the functional renormalisation group (FRG), for QCD-related reviews see [11 18]. Recent developments in FRG applications to QCD, and in particular the embedding of low energy effective models in first principle QCD can be found in 16, 19 26, for a recent survey see [27. Baryon number fluctuations and general higher moments have been studied with the FRG in $[28$ 36], see [37-42] for corresponding mean field results and interesting algorithmic developments.

Here we build on the progress made in QCD-embedded low energy effective model, see [16, 21 24, to improve on the existing fluctuations studies within an advanced FRG study of these observables: the momentum scale dependence of the quark-meson scattering is taken into account as well as the non-trivial dispersions of both quarks and mesons. Furthermore the backreaction of the matter sector on the glue dynamics is included, leading to a temperature-dependence modification of the Polyakov loop potential.

The paper is organized as follows. In Section[II the embedding of low energy effective models in QCD within the FRG framework is recalled. In Section III the QM model is discussed. The significance of the additional matter fluctuations considered in the present work is most cleanly seen without the Polyakov loop potential. We derive the flow equations for the couplings, and present numerical results on the phase structure, thermodynamics and baryon number fluctuations. In Section IV] we discuss the QCD-enhanced PQM model. This includes a discription of the QCD-enhancement of the Polyakov loop potential, as well as an discussion of the intricacies of the relation between color confinement and hadronic properties. At the end of this Section our final results are presented for the order parameter, the thermodynamics and the higher moments including a $2+1$ flavour estimate for the kurtosis in comparison to lattice data. Some useful formulae, such as threshold functions, flow equations, and further discussions can be found in the Appendix. Notably, in Appendix $\mathrm{C}$ we discuss the frequency and chemical potential dependence of fermionic couplings. This is done in view of the silver blaze property, and the thermal decay properties specifically relevant for the thermodynamics at low temperatures. 


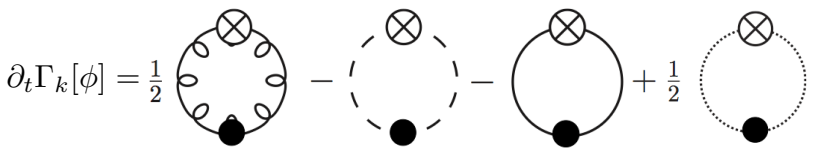

FIG. 1. Flow equation for the effective action or grand potential $\Gamma$ or $\Omega$ in full QCD. The first two loops with gluons and ghosts give the glue contribution $\Omega_{\text {glue }}$, the quark and mesonic loops give the matter contributions $\Omega_{\text {matt }}$.

\section{FROM QCD TO LOW ENERGY EFFECTIVE MODELS}

It has been argued, see e.g. 27, that low energy effective models can be derived systematically from QCD allowing for a precise determination of the fundamental model parameters. Within the functional renormalisation group framework this can be discussed already on the level of the flow equation for the scaledependent effective action, $\Gamma_{k}[\Phi]$, with the super-field $\Phi=\left(A_{\mu}, c, \bar{c}, q, \bar{q}, \phi, \ldots\right)$ with $\phi=(\sigma, \vec{\pi})$ and possible furthe effective hadronic fields. The flow equation reads schematically

$$
\partial_{t} \Gamma_{k}[\Phi]=\frac{1}{2} \operatorname{Tr} G_{\Phi \Phi}[\Phi] \partial_{t} R_{k}^{\Phi}, \quad t=\ln (k / \Lambda),
$$

where

$$
G_{\Phi_{i} \Phi_{j}}[\Phi]=\left(\frac{1}{\frac{\delta^{2} \Gamma_{k}[\Phi]}{\delta \Phi^{2}}+R_{k}^{\Phi}}\right)_{i j},
$$

is the full field-dependent propagator, $k$ is the infrared cutoff scale, and $\Lambda$ is some reference scale. The flow equation (1) is depicted in Fig. 1. for more details and QCD results, see e.g. 20, 25]27. All loops in the flow carry only momenta $q^{2} \lesssim k^{2}$ with the infrared cut-off scale $k$, and (1) implements a successive integration of momentum modes. The first two terms in Fig. 1 1 constitute the contributions of the glue system, while the third term entails the quark fluctuations and the last loop stands for the fluctuations of the effective hadronic degrees of freedom. Hence, the effective action can be written as

$$
\Gamma_{k}[\Phi]=\Gamma_{\text {glue }, k}[\Phi]+\Gamma_{\text {matt }, k}[\Phi], \quad \Gamma_{\text {matt }, k}=\Gamma_{q, k}+\Gamma_{\phi, k},
$$

where $\Gamma_{\text {glue }, k}$ includes the integrated flow of gluon and ghost loop, $\Gamma_{q, k}[\Phi]$ that of the quark loop, and $\Gamma_{\phi, k}[\Phi]$ that of the hadronic degrees of freedom. Note, that the split in quark and hadronic contributions does not reflect an effective theory setup. Within the framework of dynamical hadronisation, introduced in [13, 43, 45], it has turned out to be a very effective and powerful parameterisation of matter fluctuations in ab initio QCD in terms of genuine quark scatterings and resonant momentum channels with hadronic quantum numbers, for applications to QCD see e.g. [25, 26].

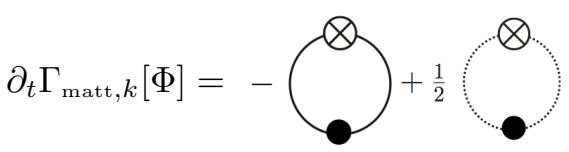

FIG. 2. Flow equation for the effective action or grand potential $\Gamma$ or $\Omega$ in the low energy regime. The gluon and quark loops in Fig. 1 are suppressed and the quark and mesonic loops give the matter contributions $\Omega_{\text {matt }}$.

Moreover, it facilitates the embedding of low energy effective theories of QCD such as NJL-type and QM-type models with and without confining Polyakov loop potential in first principle QCD: For the sake of definiteness we restrict ourselves to Landau gauge QCD. There, the physical mass gap of QCD is reflected in a mass gap in the gluon propagator of about $1 \mathrm{GeV}$. Accordingly, glue fluctuations decouple below this scale. The ghost has no mass gap but only couples to QCD matter via the gluon. Hence, it effectively shares the gluon decoupling. In summary, the fluctuations of the glue sector decouple from the matter sector in the infrared. Still, the effective potential of the glue sector, $\Omega_{\text {glue }}=\Gamma_{\text {glue }}$, is required for solving the quantum equations of motion for low energies. There, it is relevant for determining the physical glue background. In summary, integrating the QCD flow down to scales $k=\Lambda$ with $\Lambda \lesssim 1 \mathrm{GeV}$ leaves us with an effective matter theory with glue background. The flow equation for this QCD-embedded low energy effective theories is given by the two matter loops in Fig. 1, see Fig. 2. The total effective action is given by (3) where $\Gamma_{\text {glue }, k}$ is an external input. Since it does not enter the flow equation of the matter part, we only need it at $k=0$.

In a flow equation approach to $\mathrm{QCD}, \Omega_{\text {glue }}$ comprises the contributions from the gluonic and ghost loop in Fig. 1. while $\Omega_{\text {matt }}=\Gamma_{\text {matt }}$ comprises that from the quark and mesonic loop in Fig. 1. The representation in terms of a flow equation facilitates the discussion of the repsective scales. Dropping $\Omega_{\text {matt }}$ the grand potential reduces to the pure glue potential with a transition temperature $T_{\text {glue }} \approx 200 \mathrm{MeV}$, see [16, 21, 22]. The glue potential (in the current approximation) features a first order phase transition, and below $T_{\text {glue }}$ the Polyakov loop expectation vanishes. The possible minor center-breaking corrections of the glue propagation due to the vacuum polarisation are neglected. We shall see, that for the present argument we can safely drop these contributions as they only increase the expectation value of the Polyakov loop.

We conclude that the non-vanishing expectation values of $L$ and $\bar{L}$ in QCD are dominantly triggered by the quark loop in $\Omega_{\text {matt }}$. The influence of the non-trivial $A_{0^{-}}$ or $L, \bar{L}$-background will be discussed at the end of this work. For the time being we concentrate on the impact of improved matter fluctuations on the higher moments. This concerns in particular the influence of the deformed dispersion that relates to non-trivial wave function renormalisations of quarks and mesons. 


\section{QUARK-MESON MODEL}

In the spirit of the arguments of the last section we first concentrate on the low energy matter quantum, thermal and density fluctuations for two-flavour QCD. These fluctuations are well-captured within a quark meson model at scales well below $1 \mathrm{GeV}$, see the reviews [15, 17] and references therein. Moreover, full QCD calculations indicate that the width of the transition region from the quark-gluon regime to the hadronic one is small. This guarantees that the effective model is applicable and reliable at low energy, see [20, 25,27]. The scale-dependent effective action of the quark-meson model is given by

$$
\begin{aligned}
\Gamma_{k}= & \int_{x}\left\{Z_{q, k} \bar{q}\left(\gamma_{\mu} \partial_{\mu}-\gamma_{0} \mu\right) q+\frac{1}{2} Z_{\phi, k}\left(\partial_{\mu} \phi\right)^{2}\right. \\
& \left.+h_{k} \bar{q}\left(T^{0} \sigma+i \gamma_{5} \vec{T} \cdot \vec{\pi}\right) q+V_{k}(\rho)-c \sigma\right\}+\cdots
\end{aligned}
$$

with $\int_{x}=\int_{0}^{1 / T} d x_{0} \int d^{3} x$, and the dots indicate higher order terms in derivatives and fields. Accordingly, 4 has to be understood in the spirit of both, a derivative expansion at low energies as well as a vertex expansion in terms of the fluctuation physics. The validity of the latter is important for having a good grip on the fluctuation physics at low energies, and is crucial for a good access to higher moments. Note that the validity of the expansion in terms of the fluctuation physics is based on the relevance ordering of fluctuating modes that propagate off-shell in the loops. This should not be confused with the relevance of higher asymptotic states in the effective action. This important distinction is at the root of the quantitative agreement for the QCD thermodynamics computed in Polyakov quark-meson models including the trace anomaly, see 22]. In turn, in approaches based on an expansion in asymptotic states a large number of the latter is necessary to have quantitative access to the full fluctuation physics.

The meson field $\phi=(\sigma, \vec{\pi})$ introduced in (4) is in the $O(4)$ representation, with $\rho=\phi^{2} / 2$. Here $k$ is the infrared (IR) cutoff scale of FRG, see Sec III A for more details; $\mu$ is the quark chemical potential. $\vec{T}$ are the $S U\left(N_{f}\right)$ generators with $\operatorname{Tr}\left(T^{i} T^{j}\right)=\frac{1}{2} \delta^{i j}$ and $T^{0}=\frac{1}{\sqrt{2 N_{f}}} \mathbb{1}_{N_{f} \times N_{f}}$. The field-dependent effective potential $V_{k}(\rho)$ is $O(4)$ invariant, and the chiral symmetry is explicitly broken by the linear term $-c \sigma$. Thus, the mass of the Pions $\vec{\pi}$ is proportional to the linear breaking parameter $c$.

The quark and meson wave function renormalisations, i.e. $Z_{q, k}$ and $Z_{\phi, k}$, are scale-, momentum-, and fielddependent. Moreover, at finite temperature $Z$ 's split into $Z^{\|}$and $Z^{\perp}$, corresponding to those longitudinal and transversal to the heat bath, respectively. The transveral ones dominate the fluctuations physics. It has also been shown, that the approximation with scale-dependent $Z$ 's already captures the effects of the non-trivial momentum

\begin{tabular}{c|c}
\hline \hline Label & Truncations \\
\hline LPA & $\partial_{t} Z_{\phi / q}=0, \partial_{t} h=0$ \\
LPA $^{\prime}$ & $\partial_{t} Z_{\phi / q} \neq 0, \partial_{t} \bar{h}=0$ \\
LPA $+h_{k}$ & $\partial_{t} Z_{\phi / q}=0, \partial_{t} h \neq 0$ \\
full & $\partial_{t} Z_{\phi / q} \neq 0, \partial_{t} h \neq 0$ \\
\hline
\end{tabular}

TABLE I. Four different truncations and their labels used in this work.

and frequency dependence for the propagators quantitatively, see 24]. Hence, as a good approximation we only keep the scale-dependence for the wave function renormalizations, and assume $Z^{\|}=Z^{\perp}$ throughout this work. Quark and meson fields interact with each other via a scale-dependent Yukawa coupling $h_{k}$ in Eq. (4). For the fermionic parameters $Z_{q}$ and $h$ a consistent treatment of the frequency- and $\mu$-dependence is crucial for the correct low temperature physics. This is discussed in detail in Appendix C. Higher order quark-mesonic interactions can be included through the dependence of $h_{k}(\rho)$ on the meson field [23], which will be discussed elsewhere.

We investigate the thermodynamics of the QM model within various truncations. This sheds light into the roles played by the different fluctuations. Here, we denote the truncation with a full effective potential and both running $Z_{k}$ 's and $h_{k}$, as 'full'. If the flows of $Z$ 's and $h$ are turned off, i.e.

$$
\partial_{t} Z_{\phi / q}=0, \quad \partial_{t} h=0
$$

with $t$ in (1) and the reference scale $\Lambda$ being the ultraviolet (UV) cutoff, the effective action in Eq. (4) is that of the local potential approximation (LPA). Apart from LPA we also investigate the $\mathrm{LPA}^{\prime}$ approximation, i.e. the LPA with running $Z$ 's, but with a constant $h$. Here we choose a constant renormalised Yukawa coupling, $\partial_{t} \bar{h}=0$ with $\bar{h}=h / Z_{\phi}^{1 / 2} Z_{q}$, for more details see the next section. A vanishing flow of $\bar{h}$ provides a good approximation to the small scale-dependence of $\bar{h}$ in full QCD, see [25, 26]. In turn, $\partial_{t} h=0$ does not work well due to the large anomalous dimension of the mesons. Another approximation is characterized by a running $h_{k}$ but constant $Z$ 's, which is denoted as LPA $+h_{k}$ in our work. In Table I we summarise the truncations studied in this work.

\section{A. Flow equations}

As discussed in detail in Section III the flow equation for the quark meson model follows from that of first principle QCD for low cutoff scales $k \lesssim 1 \mathrm{GeV}$, where the gluonic fluctuations decouple. Then the matter loops carry the low energy fluctuations in the presence of a glue background. The glue sector only gives rise to a background potential for $A_{0}$ or the Polyakov loop $L, \bar{L}$ respectively. Dropping (the flow of) the background potential and restricting ourselves for the time being to trivial backgrounds $A_{0}=0$ or $L, \bar{L}=1$ we arrive at the flow 
equation for the quark meson model, see Fig. 2,

$$
\partial_{t} \Gamma_{k}[\Phi]=\frac{1}{2} \operatorname{Tr} G_{\phi \phi}[\Phi] \partial_{t} R_{k}^{\phi}-\operatorname{Tr} G_{q \bar{q}}[\Phi] \partial_{t} R_{k}^{q}
$$

where the super field now only includes the fluctuating quark and meson fields, $\Phi=(q, \bar{q}, \phi)$, and the regulator $R^{\Phi}=\left(R_{k}^{q}, R_{k}^{\phi}\right)$ suppresses infrared fluctuations of quark and meson fields respectively. The traces in Eq. (6) sum over momenta and internal quark (Dirac and color) and meson (flavour) indices. The full, field-dependent propagator, 2 reduces to the coupled one of quarks and mesons, to wit,

$$
G_{\phi \phi / q \bar{q}}[\Phi]=\left(\frac{1}{\frac{\delta^{2} \Gamma_{k}[\Phi]}{\delta \Phi^{2}}+R_{k}^{\Phi}}\right)_{\phi \phi / q \bar{q}},
$$

for the diagonal parts that show up in (6).

\section{B. Flow equations for the effective potential}

In this work we use $3 d$ flat regulators, [46, 47, for quarks and mesons, see also Appendix A. The flow of the effective potential $V_{k}(\rho)$ is obtained by substituting Eqs. (4), (7) in (6) and evaluating the flow at constant mesonic fields,

$$
\begin{aligned}
\partial_{t} V_{k}(\rho)= & \frac{k^{4}}{4 \pi^{2}}\left[\left(N_{f}^{2}-1\right) l_{0}^{(B, 4)}\left(\bar{m}_{\pi, k}^{2}, \eta_{\phi, k} ; T\right)\right. \\
& +l_{0}^{(B, 4)}\left(\bar{m}_{\sigma, k}^{2}, \eta_{\phi, k} ; T\right) \\
& \left.-4 N_{c} N_{f} l_{0}^{(F, 4)}\left(\bar{m}_{q, k}^{2}, \eta_{q, k} ; T, \mu\right)\right]
\end{aligned}
$$

where $l_{0}^{(B / F, 4)}$, e.g. [20, 23], are the threshold functions, see Appendix A. The renormalised dimensionless meson and quark masses are given by

$$
\begin{aligned}
& \bar{m}_{\pi, k}^{2}=\frac{V_{k}^{\prime}(\rho)}{k^{2} Z_{\phi, k}} \\
& \bar{m}_{\sigma, k}^{2}=\frac{V_{k}^{\prime}(\rho)+2 \rho V_{k}^{\prime \prime}(\rho)}{k^{2} Z_{\phi, k}} \\
& \bar{m}_{q, k}^{2}=\frac{h_{k}^{2} \rho}{2 k^{2} Z_{q, k}^{2}}
\end{aligned}
$$

and the anomalous dimensions are defined by

$$
\eta_{\phi, k}=-\frac{\partial_{t} Z_{\phi, k}}{Z_{\phi, k}}, \quad \eta_{q, k}=-\frac{\partial_{t} Z_{q, k}}{Z_{q, k}} .
$$

Here we consider frequency and spatial momentumindependent anomalous dimension, the anomalous dimensions are evaluated at low frequencies and spatial momenta. The related depencence if covered by the $k$ dependence. The precise definition of the anomalous dimensions in 10 and a detailed discussion of this approximation is found in Appendix $\mathrm{B}$ for $\eta_{\phi}$, and in Appendix $\mathrm{C}$ for $\eta_{q}$. The latter also contains an evaluation of the $\mu$ and $T$ dependence important for the silver blaze property of QCD and the thermodynamics at low temperatures.

In general it is more convenient to work with renormalized fields and renormalization group ( $R G$ )-invariant quantities. We denote them by symbols with bar, as the renormalized masses shown in Eqs. (9). The relations between renormalised quantities and original ones read

$$
\bar{\phi}=Z_{\phi, k}^{\frac{1}{2}} \phi, \quad \bar{h}_{k}=\frac{h_{k}}{Z_{q, k} Z_{\phi, k}^{\frac{1}{2}}},
$$

taking the meson field and Yukawa coupling as examples. Thus we have $\bar{\rho}=Z_{\phi, k} \rho$ and the effective potential $\bar{V}_{k}(\bar{\rho})=V_{k}(\rho)$. Note that the chiral symmetry breaking term $-c \sigma$ in Eq. (4) is linear in the fields. Hence it neither contributes to the right hand side of any flow, nor does it flow. Considering $\bar{c}_{k}=c / Z_{\phi, k}^{1 / 2}$, this leads to

$$
\partial_{t} \bar{c}_{k}=\frac{1}{2} \eta_{\phi, k} \bar{c}_{k}
$$

In the present work, we solve the flow equation for the effective potential, Eq. (8), within a Taylor expansion about a fixed unrenormalised field value $\kappa$, to be contrasted to one about the scaling minimum $\rho_{0, k}$, see e.g. 48. It has been shown in [23] that such a Taylor expansion about a fixed $\kappa$ has the most rapid convergence. Expanded in renormalised fields, the effective potential then reads

$$
\bar{V}_{k}(\bar{\rho})=\sum_{n=0}^{N} \frac{\bar{\lambda}_{n, k}}{n !}\left(\bar{\rho}-\bar{\kappa}_{k}\right)^{n},
$$

with $\bar{\lambda}_{n, k}=\lambda_{n, k} / Z_{\phi, k}^{n}$ and $\bar{\kappa}_{k}=Z_{\phi} \kappa$ with

$$
\partial_{t} \bar{\kappa}_{k}=-\eta_{\phi, k} \bar{\kappa}_{k}
$$

In accordance with the convergence discussion in 23 we already find a good convergence for $N=5$. For example, the difference for $f_{\pi}=\bar{\sigma}$ between $N=5$ and $N=6,7$ is less than $1 \%$ for all temperatures. Consequently we use $N=5$ for all computations. Inserting Eq. 13 into the left hand side of Eq. (8), one arrives at

$$
\begin{aligned}
& \left.\partial_{\bar{\rho}}^{n}\left(\left.\partial_{t}\right|_{\rho} \bar{V}_{k}(\bar{\rho})\right)\right|_{\bar{\rho}=\bar{\kappa}_{k}} \\
= & \left(\partial_{t} \bar{\lambda}_{n, k}-n \eta_{\phi, k} \bar{\lambda}_{n, k}\right)-\left(\partial_{t} \bar{\kappa}_{k}+\eta_{\phi, k} \bar{\kappa}_{k}\right) \bar{\lambda}_{n+1, k},
\end{aligned}
$$

Note that the term proportional to the higher order coupling $\bar{\lambda}_{n+1, k}$ vanishes due to Eq. 14. This is at the root of the rapid convergence of the present expansion scheme, see 23. In our computation the expansion point is chosen such that $\bar{\kappa}_{k=0}=\bar{\rho}_{0, k=0}$. Hence it is an expansion at the physical minimum, and we are well within the convergence radius of such an expansion.

Apart from the flow equation for the effective potential, we also need the flow equations of the Yukawa coupling, and the anomalous dimensions. They are deferred to the Appendix C. 

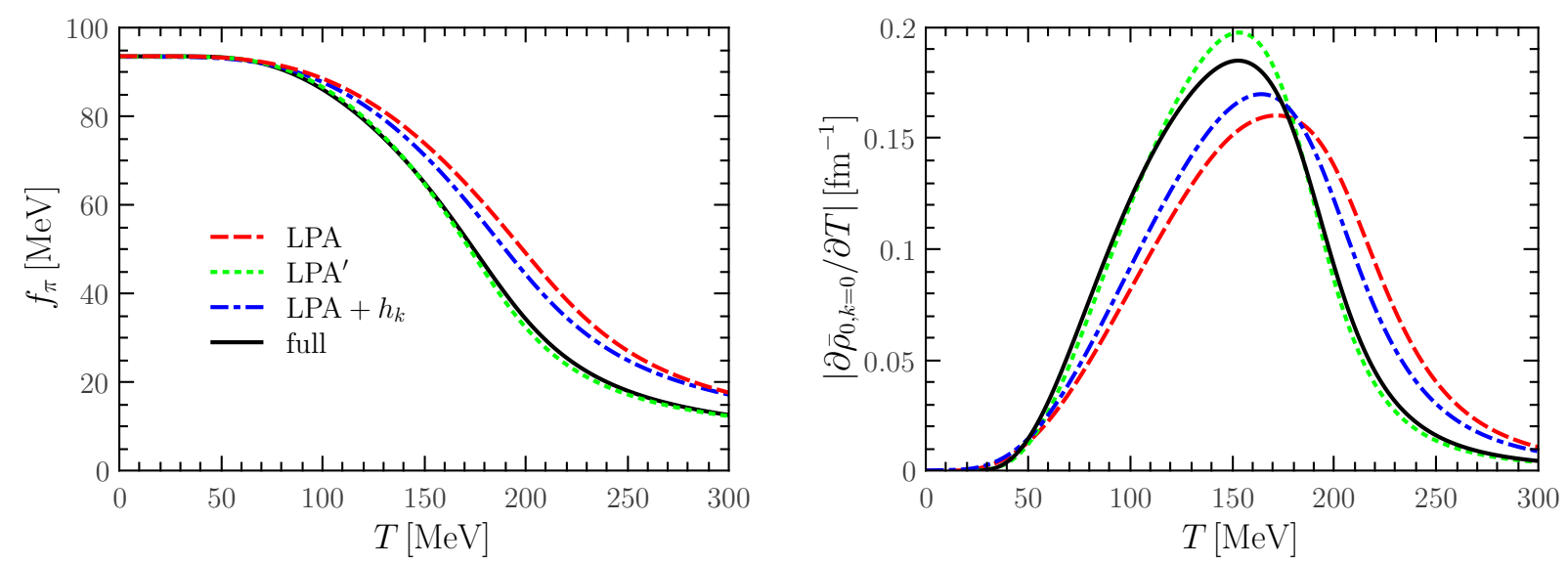

FIG. 3. Order parameter of the chiral phase transition: $f_{\pi}=\left(2 \bar{\rho}_{0, k=0}\right)^{1 / 2}$ (left panel), and $\left|\partial \bar{\rho}_{0, k=0} / \partial T\right|($ right panel) as functions of the temperature in the different truncations of Table I.

\section{Initial conditions}

It is left to specify the input parameters of the effective model. Within the FRG approach, this is done by fixing the initial effective action $\Gamma_{\Lambda}$ in the vacuum. The UVcutoff scale is chosen to be $\Lambda=700 \mathrm{MeV}$ throughout this work.

Choosing a larger $\Lambda$ requires the inclusion of dynamical hadronisation as well as that of gluonic fluctuations: the validity range of the current model can be accessed in the vacuum by using the results of the QCD flows in [25, 26. at a given cutoff scale $k=\Lambda$ as initial effective action of the model at hand. The results of such a computation can then be compared with the results of the QCD flows. The larger the initial cutoff scale $\Lambda$ is, the larger the deviations grow. This limits the reliability of the model predictions.

In turn, choosing a smaller $\Lambda$ limits our thermal range, as the physics of temperatures with $T / \Lambda \gtrsim 1 / 7-1 / 5$ is severely contaminated by cutoff effects, see [24]. This entails that the maximal temperature accessible within the current model is $T_{\max }=100-140 \mathrm{MeV}$. In the present work we also amend the model with temperaturedependent initial conditions which gives access to higher temperatures. This is detailed in the next Section IIID.

At the initial scale the effective potential is well approximated by a classical potential, to wit

$$
\bar{V}_{\Lambda}(\bar{\rho})=\frac{\bar{\lambda}_{\Lambda}}{2} \bar{\rho}^{2},
$$

\begin{tabular}{c|c|c|c|c}
\hline \hline Truncations & $\bar{\lambda}_{\Lambda}$ & $\bar{h}_{\Lambda}$ & $\bar{c}_{\Lambda}\left(\times 10^{-3} \mathrm{GeV}^{3}\right)$ & $m_{\sigma}(\mathrm{MeV})$ \\
\hline LPA & 43 & 6.5 & 1.7 & 585.3 \\
LPA $^{\prime}$ & 72 & 6.5 & 1.98 & 584.7 \\
LPA $+h_{k}$ & 36.8 & 5.5 & 1.7 & 551.2 \\
full & 77 & 7.1 & 2.0 & 578.9 \\
\hline
\end{tabular}

TABLE II. Input parameters and the predicted $\sigma$-meson mass with different truncations. with the relevant coupling $\bar{\lambda}_{\Lambda}=\bar{\lambda}_{2, \Lambda}$. For the sake of simplicity we have chosen the mass $m_{\phi, \Lambda}^{2}=0$. This fixes the $\sigma$-mass and is respsonsible for its minor variation for the different truncations as well as a relatively large meson scattering coupling $\bar{\lambda}_{\Lambda}$ at the initial scale, see Table II. Heuristically, if aiming at maximising the effective thermal and chemical potential range one has to utilise the meson mass parameter in order to minimise $\bar{\lambda}_{\Lambda}$. Such a choice leads to small mesonic contributions at large cutoff scales $k \rightarrow \Lambda$ and hence at large $T$ and $\mu$. This simulates the melting of the mesons at large scales. Still, it rather is a feature of the model instead of built-in physics. In the present framework the melting of mesons is naturally induced by dynamical hadronisation and will be considered elsewhere. Here, we stick to (16).

The other two relevant couplings are the Yukawa coupling $\bar{h}_{\Lambda}$ and the coefficient of the linear breaking term $\bar{c}_{\Lambda}$. The three relevant couplings of the model are determined by fitting hadronic observables in the vaccum, the $\pi$ decay constant $f_{\pi}=\bar{\sigma}$ with $f_{\pi}=93.5 \mathrm{MeV}$, the $\pi$-meson mass $m_{\pi}=135 \mathrm{MeV}$, and the quark mass $m_{q}=1 / 2 \bar{h} \bar{\sigma}$ with $m_{q}=303.5 \mathrm{MeV}$. The three couplings at the initial scale $k=\Lambda$ as well as the predicted $\sigma$-meson mass at vanishing cutoff $k=0$ are summarised in Table II for the different truncations discussed in Section III.

\section{Pressure and entropy}

The thermodynamical potential density, i.e. grand potential density, $\Omega_{k}(\bar{\rho})$ is related to the effective potential through

$$
\Omega_{k}(\bar{\rho})=\Gamma_{k, T}[\bar{\rho}]-\Gamma_{k, T=0}[\bar{\rho}]
$$

which is normalised to zero at vanishing temperature. It has been argued above that the thermal range is given by $\Lambda / T_{\max } \approx 5-7,24$. The ultraviolet cutoff $\Lambda$ used in the present work has been chosen as $\Lambda=700 \mathrm{MeV}$ in order to minimise the systematic errors of missing glue fluctuations and additional four-fermi and other higher order 

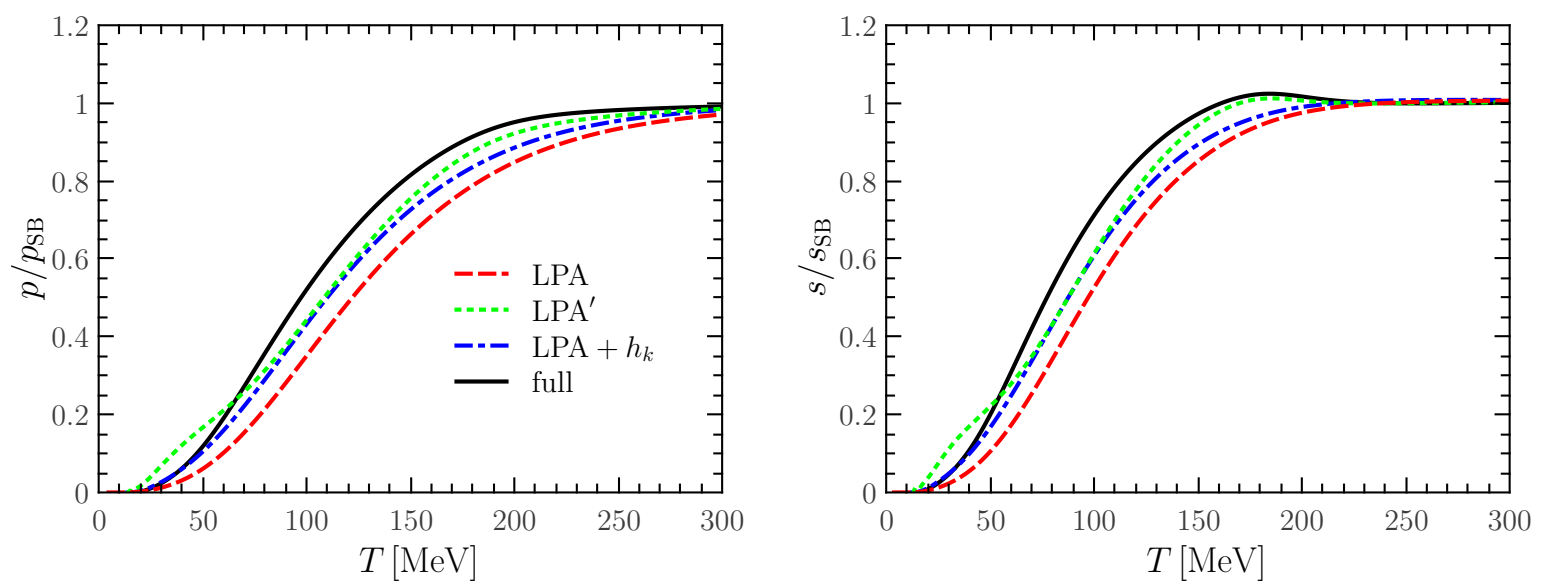

FIG. 4. Pressure (left panel) and entropy density (right panel), normalised to their Stefan-Boltzmann values, as functions of $T$.

couplings that can be described within dynamical hadronisation. However, then temperature-dependent initial conditions are required for $T \gtrsim 100-140 \mathrm{MeV}$ : Already the flow at the initial scale is sensitive to thermal fluctuations for these temperatures, and hence the initial action as well as thermodynamical quantities, such as the pressure, entropy, fluctuations etc. are. This has been discussed at length in [22, where it has been used for a systematic error estimate. In the present case the thermodynamical potential density at $\Lambda$ can be estimated by the integrated flow of the difference in (17) without feeding back the flow of the parameters. This leads to

$$
\begin{aligned}
\Omega_{\Lambda} \approx-\frac{4 N_{c} N_{f}}{12 \pi^{2}} \int_{\Lambda}^{\infty} d k k^{3} & {\left[\frac{1}{\exp \left(\frac{k-\mu}{T}\right)+1}\right.} \\
& \left.+\frac{1}{\exp \left(\frac{k+\mu}{T}\right)+1}\right],
\end{aligned}
$$

where we have dropped the negligible contribution of the mesonic degrees of freedom for cutoff scales above the chiral symmetry breaking scale $k_{\chi} \ll \Lambda$. We estimate that this procedure effectively extends the thermal range to $T \lesssim \Lambda / 5-\Lambda / 3$, as it mimics the thermal change of the initial condition at $k=\Lambda$. For the ultraviolet cutoff $\Lambda=700 \mathrm{MeV}$ used here this gives us access to temperatures with $T_{\max } \approx 140-230 \mathrm{MeV}$. The corresponding systematic error estimates from [22] will be used later for an error estimate of the bayronic fluctuations.

The pressure and entropy density follow readily as

$$
p=-\Omega_{k=0}\left(\bar{\rho}_{0, k=0}\right), \quad \text { and } s=\frac{\partial p}{\partial T},
$$

from the grand potential $\Omega$. Fig. 3 (left panel) compares the temperature dependence of the order parameter $f_{\pi}=\bar{\sigma}$ on $T$ in different truncations. One observes that the truncations cluster into two groups: full and $\mathrm{LPA}^{\prime}$ in one group, LPA and LPA $+h_{k}$ in the other. The crossovers in LPA and LPA $+h_{k}$ are broader than that of full approximation and LPA'. This entails that the non-trivial momentum dependence of the propagators, encoded in the wave function renormalization factors, increases the strength of the crossover and lowers the pseudo-critical temperature. This is seen very clearly in the right panel of Fig. 3 where $\left|\partial \bar{\rho}_{0, k=0} / \partial T\right|$ as a function of temperature is depicted. We also expect larger changes in $\mathrm{LPA}^{\prime}$ and full approximation for the high-order fluctuations, see Section IIIE,

In Fig. 4 we show the pressure and entropy density as functions of the temperature in different truncations. We have normalised them with those of massless ideal gas of quarks, i.e. the Stefan-Boltzmann (SB) limit,

$$
\frac{p_{\mathrm{SB}}}{T^{4}}=2 N_{c} N_{f}\left[\frac{7 \pi^{2}}{360}+\frac{1}{12}\left(\frac{\mu}{T}\right)^{2}+\frac{1}{24 \pi^{2}}\left(\frac{\mu}{T}\right)^{4}\right]
$$

One observes that the pressures calculated with truncations full and $\mathrm{LPA}^{\prime}$ increase with $T$ more rapidly than those with the other two truncations, and their values are also larger. These findings indicate that propagators with improved momentum-dependence stiffen the equation of state. A similar behavior is found in the calculations of entropy density, as shown in the right panel of Fig. 4. The entropy obtained with wave function renormalization factors rises more rapidly.

In approximations with scale-dependent wave function renormalisations both the entropy as well as quadratic baryonic fluctuation show a peak at about $170-180 \mathrm{MeV}$. This is an artefact of the current approximation, and can be traced back to the systematic errors discussed before. First of all these temperatures are above the estimate from 24 for the maximal temperature $T_{\max } \approx 100-140$ $\mathrm{MeV}$ as well as in the border regime of the enhanced estimate with $T_{\max } \approx 140-230 \mathrm{MeV}$. Hence they are subject to cutoff effects. Moreover, we already know from QCD flows at vanishing temperature that the present local approximation with scale-dependent wave function renormalisations without full momentum dependence overestimates the anomalous dimensions in the scale regime 

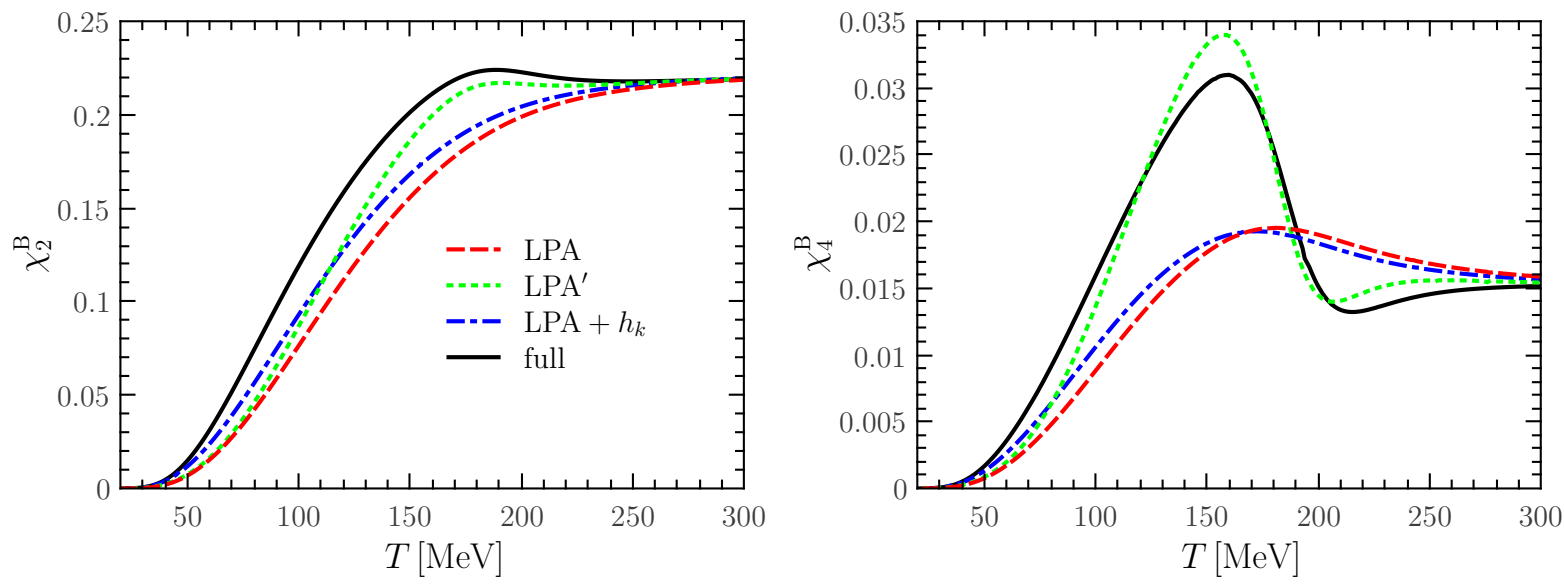

FIG. 5. Quadratic (left panel) and quartic (right panel) baryon number fluctuations as functions of $T$ in the QM model for the different truncations, see Table 1

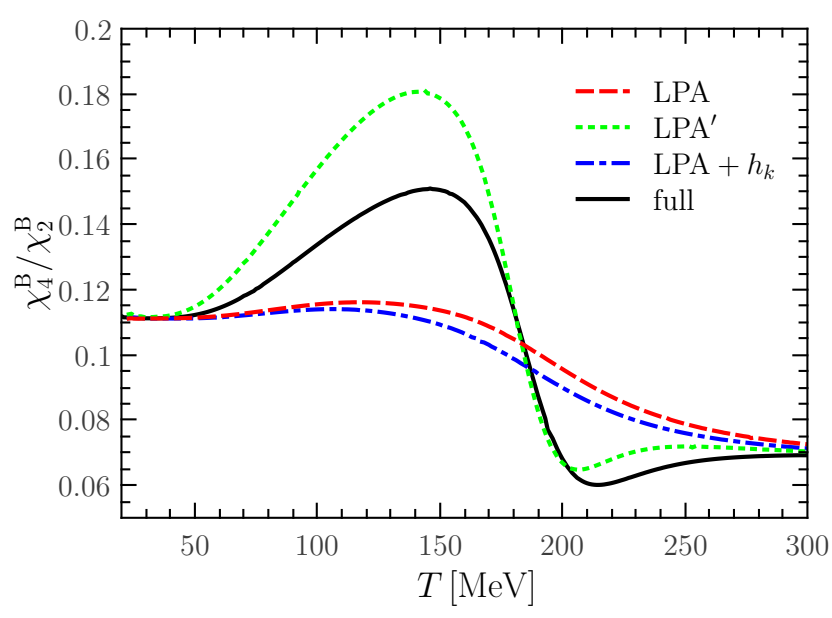

FIG. 6. Kurtosis of the baryon number distribution as a function of the temperature in the QM model.

slightly above the chiral scale, see [25] and [26] for a comparison of the scale and momentum-dependence. A related comparison concerning the scale dependent running strong coupling with and without momentum dependence can also be found in [49, see Fig. 12. This calls for a refined analysis with momentum and frequency dependent propagators which will be considered elsewhere.

Finally, we remark that the $\mathrm{LPA}^{\prime}$ and full approximation leads to a very small negative pressure for small temperatures $T \lesssim 80 \mathrm{MeV}$, see the inlay plot in Fig. 15 . Its origin can be traced back to the anomalous dimension of the qaurk, and hints at a -minor- overestimation of thermal flucutation effects of the quark propagation for constant anomalous dimensions. We expect this effect to disappear once the full frequency and momentum dependence of the propagator is taken into account. In any case, it is a very minor effect, as shown in Fig. 15. and only exists the low-temperature regime far below the pseudo-critical temperature.

\section{E. Baryon number fluctuations}

The baryon number fluctuations are usually described by the generalized susceptibilities,

$$
\chi_{n}^{\mathrm{B}}=\frac{\partial^{n}}{\partial\left(\mu_{\mathrm{B}} / T\right)^{n}} \frac{p}{T^{4}},
$$

with baryon number chemical potential $\mu_{\mathrm{B}}=3 \mu \cdot \chi_{n}^{\mathrm{B}}$ 's are related with the cumulants of baryon multiplicity distributions. Denoting the ensemble average of the baryon number with $\left\langle N_{\mathrm{B}}\right\rangle$, the quadratic and quartic fluctuations are given by

$$
\begin{aligned}
\chi_{2}^{\mathrm{B}} & =\frac{1}{V T^{3}}\left\langle\delta N_{\mathrm{B}}{ }^{2}\right\rangle, \\
\chi_{4}^{\mathrm{B}} & =\frac{1}{V T^{3}}\left(\left\langle\delta N_{\mathrm{B}}{ }^{4}\right\rangle-3\left\langle\delta N_{\mathrm{B}}{ }^{2}\right\rangle^{2}\right),
\end{aligned}
$$

where $V$ is the volume of the system, and $\delta N_{\mathrm{B}}:=N_{\mathrm{B}}-$ $\left\langle N_{\mathrm{B}}\right\rangle$. In fact, $\left\langle\delta N_{\mathrm{B}}{ }^{2}\right\rangle$ is just the width of the Gaussian distribution, denoted specifically by $\sigma^{2}$. The kurtosis of non-Gaussian distributions $\kappa$ (the skewness is vanishing at $\left.\mu_{\mathrm{B}}=0\right)$ is defined by

$$
\kappa \sigma^{2}=\frac{\chi_{4}^{\mathrm{B}}}{\chi_{2}^{\mathrm{B}}}
$$

The quadratic baryon number fluctuation $\chi_{2}^{\mathrm{B}}$ as a function of $T$ with different truncations is presented in left panel of Fig. 5. As expected, $\chi_{2}^{\mathrm{B}}$ in LPA and LPA $+h_{k}$ signal a broad crossover, while in the other two truncations show a steeper transition. In general, the $\chi_{2}^{\mathrm{B}}$ behave very similar to $s / s_{\mathrm{SB}}$ shown in Fig. 4 .

So far, we have compared the pressure and its loworder derivatives with respect to the temperature ( $s$ : first order) and chemical potential $\left(\chi_{2}^{\mathrm{B}}\right.$ : second order) within different truncations. All the comparisons indicate that the nontrivial momentum dependence of propagators enhances the transition strength of the chiral crossover. 
This effect should be even more pronounced in higherorder thermodynamical quantities, such as $\chi_{4}^{\mathrm{B}}$. This expectation is confirmed in Fig. 5 , right panel and Fig. 6 . where $\chi_{4}^{\mathrm{B}}$ and the kurtosis $\kappa \sigma^{2}$ are shown. Both $\chi_{4}^{\mathrm{B}}$ and $\kappa \sigma^{2}$, calculated in the full and $\mathrm{LPA}^{\prime}$ approximations, vary rapidly during the crossover. In contradistinction, those obtained from LPA and LPA $+h_{k}$ as functions of $T$ are much smoother. Note that the difference is quite large in the quartic fluctuations, in contrast to that observed in low-order thermodynamical quantities.

In light of an experimental observation of (critical) fluctuations this is good news. It has been shown previously, that effects of mesonic multi-scattering in the medium, as included in the FRG approach in a LPA truncation, soften the chiral crossover and suppress thermal fluctuations compared with the mean-field approximation, see e.g. 28 for the kurtosis. This limits the chances of an experimental observation of critical fluctuations and hence the critical point. However, our present calculations reveal that the nontrivial quantum and thermal dispersions of quarks and mesons have the opposite effect: they increase the transition strength of the crossover and enhances thermal fluctuations. While this effect was already visible in 23, 24. for the order parameter, it is particularly obvious for high-order thermal fluctuations, such as non-Gaussian fluctuations of the baryon number, see Fig. 6 .

\section{QCD-ENHANCED POLYAKOV-QUARK-MESON MODEL}

The quark-meson model studied so far is a good and clean testing ground for studying the relative strength of flcutuation effects such as multi-meson scattering and non-trivial dispersions. However, it does not include the confinement dynamics that is necessary to obtain the correct physics of baryon number fluctuations in the hadronic regime at temperatures below $T_{c}$.

As already argued in Section II the quark-meson model can be embedded in low energy/low temperature QCD by adding a background gluonic potential, see (3). This directly leads to the usual Polyakov-loop-extended chiral models, such as the Polyakov-Nambu-Jona-Lasinio model [50 52] and the Polyakov-quark-meson (PQM) model [53]. As explained in Section II, at sufficiently low scales the gluon fluctuations decouple due to the QCD mass gap, and the gluon field can be treated as a temporal background field. Its contribution to the thermodynamics, however, is prominent, via the expectation value of the traced Polyakov loop, viz.

$$
L(\vec{x})=\frac{1}{N_{c}}\langle\operatorname{Tr} \mathcal{P}(\vec{x})\rangle, \quad \text { and } \quad \bar{L}=\frac{1}{N_{c}}\left\langle\operatorname{Tr} \mathcal{P}^{\dagger}(\vec{x})\right\rangle,
$$

with

$$
\mathcal{P}(\vec{x})=\mathcal{P} \exp \left(i g \int_{0}^{\beta} d \tau A_{0}(\vec{x}, \tau)\right) .
$$

The expectation value of the traced Polyakov loop, $L, \bar{L}$ serve as order parameters for the deconfinement phase transition.

In the presence of a gluonic background field the quark contribution to the flow is changed. This is accounted for by simply replacing the fermion distribution functions $n_{F}(x, T)=1 /(\exp (x / T)+1)$ in Appendix A with the Polyakov-loop modified ones, i.e.

$$
n_{F}(x, T, L, \bar{L})=\frac{1+2 \bar{L} e^{x / T}+L e^{2 x / T}}{1+3 \bar{L} e^{x / T}+3 L e^{2 x / T}+e^{3 x / T}} .
$$

In $(26)$ we have introduced the notation

$$
\begin{aligned}
& x=\frac{k}{z_{q}}\left(1+\bar{m}_{q, k}^{2}\right)^{1 / 2}-\mu, \\
& \bar{x}=\frac{k}{z_{q}}\left(1+\bar{m}_{q, k}^{2}\right)^{1 / 2}+\mu,
\end{aligned}
$$

with $z_{q}=1$, see Appendix A. The distribution function of the anti-quark, $n_{F}(\bar{x}, T)$ changes to

$$
n_{F}(\bar{x}, T) \rightarrow n_{F}(\bar{x}, T, \bar{L}, L) .
$$

In this work we present results in two different approximations. In the first approximation we aim at exploiting the existing results of the Polyakov loop expectation value, e.g. from the lattice or continuum QCD computations. Then the Polyakov loop expectation value serve as a background in which the flow of the matter fluctuations is computed. Furthermore, $\bar{L}=L$ is assumed and their dependence on the small chemical potential is neglected. Here we simply test the consistency of such an approach as well as evaluating the sensitive input parameters such as the field derivatives of the Polyakov loop potential. The respective results in this approach are collected in Appendix E.

The second approximation utilises a given Polyakov loop potential, and the equations of motion for $L$ and $\bar{L}$ are solved together with that of the $\sigma$-meson. The matter fluctuations are computed on this self-consistent background, leading to a fully coupled system. This approach is numerically more challenging, in particular if it comes to extensions of the present approacimation. However, it has the advantage of self-consistency which turns out to be of eminent importance for the computation of baryonic fluctuations, or higher moments in general.

\section{A. Glue potential and higher moments}

Now we introduce the simple polynomial Polyakov loop model potential $\mathcal{U}(L, \bar{L})$, see [51]. We shall also discuss the model parameters relevant for a reliable extraction of higher moments in QCD. A more thorough investigation of the model dependence of the results will be considered elsewhere. The dimensionless Polyakov-loop potential $V_{\mathrm{YM}}[L, \bar{L} ; t]$ is obtained by a rescaling of $\mathcal{U}(L, \bar{L})$ with 
$1 / T^{4}$. It can be expressed in terms of the dimensionless reduced temperature $t=\left(T-T_{\text {cr }}\right) / T_{\text {cr }}$ and reads

$$
\begin{aligned}
V_{\mathrm{YM}}(L, \bar{L} ; t) & =\frac{\mathcal{U}(L, \bar{L})}{T^{4}} \\
& =-\frac{b_{2}(t)}{2} L \bar{L}-\frac{b_{3}}{6}\left(L^{3}+\bar{L}^{3}\right)+\frac{b_{4}}{4}(L \bar{L})^{2} .
\end{aligned}
$$

Only the coefficient $b_{2}(t)$ of the quadratic term depends on temperature. For large temperatures we have $b_{2}>0$, while for small ones we have $b_{2}<0$. Its temperature dependence above $T_{c}$ is fixed by the Yang-Mills expectation value of the Polyakov loop computed on the lattice and the Yang-Mills pressure. It has a divergence for $t \rightarrow-1$, that is for vanishing temperature $T \rightarrow 0$.

$$
b_{2}(t)=a_{0}+\frac{a_{1}}{1+t}+\frac{a_{2}}{(1+t)^{2}}+\frac{a_{3}}{(1+t)^{3}},
$$

with the parameters $a_{0}=6.75, a_{1}=-1.95, a_{2}=2.625$, $a_{3}=-7.44, b_{3}=0.75, b_{4}=7.5$. Our computations here are based on a QCD-enhanced glue potential, that has the correct thermal dependence on the reduces temperature of QCD instead that of Yang-Mills theory. It has been shown in [16, 21, 22 that in the vicinity of the phase transition the thermal QCD scaling is obtained from that in Yang-Mills theory in (29) through a simple linear rescaling of $t$,

$$
t_{\text {YM }}\left(t_{\text {glue }}\right) \approx 0.57 t_{\text {glue }} .
$$

Thus, the QCD-enhanced glue potential reads

$$
V_{\text {glue }}\left(L, \bar{L} ; t_{\text {glue }}\right)=V_{\text {YM }}\left(L, \bar{L} ; 0.57 t_{\text {glue }}\right),
$$

with $t_{\text {glue }}=\left(T-T_{\mathrm{cr}}^{\text {glue }}\right) / T_{\mathrm{cr}}^{\text {glue }}$ and $T_{\mathrm{cr}}^{\text {glue }} \approx 200-210 \mathrm{MeV}$ for two flavours [22]. Here we choose $208 \mathrm{MeV}$ as already used in 53 .

The QCD enhancement of the potential provides the correct temperature scaling of the glue potential in QCD. QCD-enhanced computations of thermodynamical quantities, i.e. pressure and trace anomaly, agree remarkably well with recent results from lattice QCD for the $N_{f}=2+1$ flavour case already in LPA, see [22. Moreover, despite the absence of the strange quark fluctuations, already the $N_{f}=2$ flavour thermodynamical observables are close to that in the $N_{f}=2+1$ case, after the overall temperature scale is normalised, for a comparison of $N_{f}=2+1$ to $N_{f}=2+1+1$ see [54]. This supports the interpretation that the strange quark (and even more so the charm quark for that matter) contribute to the UV fluctuations above their mass scale and hence predominantly only change the overall dynamical scale $\Lambda_{\mathrm{QCD}}$. In turn, at low momentum and temperature scales the strange quark decouples and its fluctuations only leads to quantitative modifications of the thermodynamical observables. In the present work we shall test this interpretation also for the baryonic fluctuations.

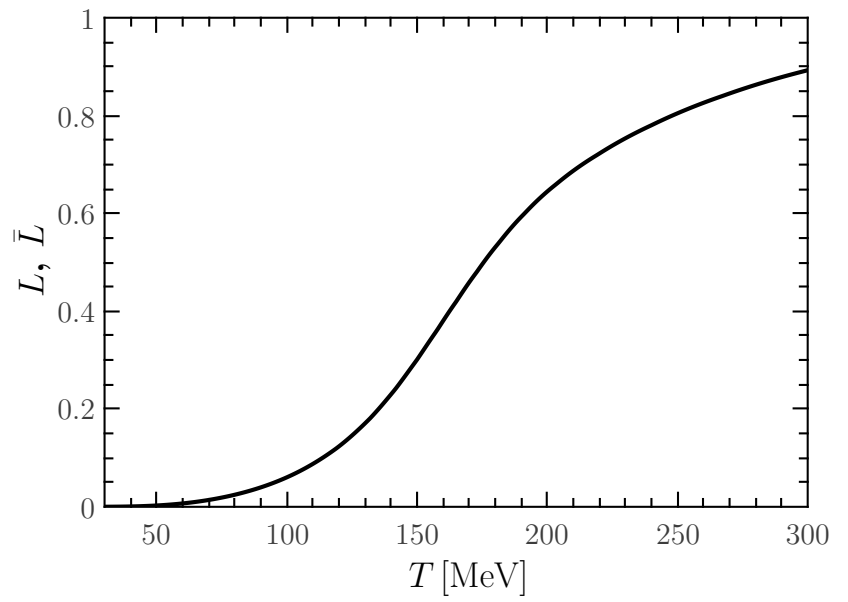

FIG. 7. Polyakov loop as a function of $T$. The result is obtained by employing the QCD-enhanced glue potential in 22 .

The respective Polyakov loop expectation values within the present self-consistent two flavour computation are depicted in Fig. 7 as a function of $T$. We emphasise again that the potential itself is a model potential that reproduces the pure glue pressure and Polyakov loop expectation values. It may fall short of reproducing higher Polyakov loop correlation functions. The latter information can e.g. be introduced to the models by computing higher order correlation functions or Polyakov loop fluctuations, see [55, 56. A full resolution of this task is given by either computing the full glue potential from functional methods, [19, 57 [59], or by computing the full potential on the lattice 60 64. The full QCDembedding with the computation of the glue potential with the FRG will be considered elsewhere.

In any case it is of chief interest to evaluate the sensitivity of the results on the higher moments of the baryon multiplicity distribution on the higher order $L, \bar{L}$ derivatives of the glue potential. We will see in the next subsection that the absolute values of the higher moments $\chi_{n}^{\mathrm{B}}$, in asymptotic $T / T_{c}$-regime, crucially depend on parameters of the glue-potential $\Omega_{\text {glue }}$. Moreover, the transition regime depends on the relative strength of the couplings in $\Omega_{\text {glue }}$ and $\Omega_{\text {matt }}$. This emphasises the necessity to compute and utilise quantitative versions of the glue potential, as well as a detailed study of the inputdependence. This is beyond the scope of the present work and shall be discussed elsewhere, 65].

Here we put forward an analytical method for the computation of higher moments that clearly demonstrate the relation between $\chi_{n}^{\mathrm{B}}$ and the $L, \bar{L}$ expansion coefficients (higher moments) of the glue potential. The higher moments $\chi_{n}^{\mathrm{B}}$ are proportional to total $\mu$-derivatives of $\Omega$. Hence, it requires the computation of $\partial_{\mu} L$ and $\partial_{\mu} \bar{L}$ from the correlation functions, that are the field derivatives of $\Omega$ at vanishing $\mu$. This rather technical computation is deferred to Appendix D here we only briefly describe the main results. 
Total $\mu$-derivatives can be written as partial $\mu$ derivatives at fixed fields and field-derivatives multiplied with $\partial_{\mu} \Phi_{i}$ with $\Phi=(L, \bar{L}, q, \bar{q}, \sigma, \vec{\pi})$, see (D9) in Appendix D.

$$
\frac{d}{d \mu}=\left.\partial_{\mu}\right|_{\Phi}-\Omega_{\mu i} G_{i j} \frac{\delta}{\delta \Phi_{j}} .
$$

Here $G_{i j}$ stands for the propagator, see (D7) and $\Omega_{\mu i}$ stands for a mixed partial $\mu$ and $\Phi_{i}$-derivative, see (D7). The indices $i, j$ sum over species of fields, momentum or space-time indices as well as internal indices. Within this condensed notation, the second moment takes the form (D8),

$$
\frac{d^{2} \Omega\left[\Phi_{\mathrm{EoM}}\right]}{d \mu^{2}}=\Omega_{\mu^{2}}-\Omega_{\mu i} G_{i j} \Omega_{\mu j} .
$$

In the low temperature limit both terms, $\Omega_{\mu^{2}}$ as well as that proportional to $\Omega_{\mu i}$ contribute. We re-order (34) and concentrate on $\chi_{2}^{\mathrm{B}}$. Note also that in the present approximation to the full theory we have $\Omega_{\mu \pi}=\Omega_{\mu q}=0$. This leads us to

$$
\chi_{2}^{\mathrm{B}} \simeq \frac{1}{9}\left(\Omega_{\mu^{2}}-\Omega_{\mu \sigma}^{2} G_{\sigma \sigma}\right)-\frac{1}{9} \Delta \Omega_{2},
$$

with

$$
\begin{aligned}
\Delta \Omega_{2}= & 2\left(\Omega_{\mu \sigma} \Omega_{\mu L} G_{\sigma L}+\Omega_{\mu \sigma} \Omega_{\mu \bar{L}} G_{\sigma \bar{L}}\right) \\
& +\Omega_{\mu L}^{2} G_{L L}+\Omega_{\mu \bar{L}}^{2} G_{\bar{L} \bar{L}}+2 \Omega_{\mu L} \Omega_{\mu \bar{L}} G_{L \bar{L}} .
\end{aligned}
$$

The term $\Delta \Omega_{2}$ carries the direct sensitivity to the model input, the choice of the Polyakov loop potential $\Omega_{\text {glue }}=$ $T^{4} V_{\text {glue }}$. Most importantly this is reflected in the propagators $G_{L L}, G_{\bar{L} \bar{L}}, G_{L \bar{L}}$ that depend in leading order on $\Omega_{\text {glue }}$. Note that also the other propagators depend on $\Omega_{\text {glue }}$ as $\Omega_{i j}^{(2)}$ is not diagonal, but these dependencies are sub-leading. In summary, (36) entails that for an accurate determination of the second moment $\chi_{2}^{\mathrm{B}}$ the glue potential used in model computations should reproduce the (connected) two-point correlation functions $G_{L L}=\langle L L\rangle_{c}, G_{L \bar{L}}=\langle L \bar{L}\rangle_{c}$, and $G_{\bar{L} \bar{L}}=\langle\bar{L} \bar{L}\rangle_{c}$ in QCD. Alternatively the subdominance of these contributions has to be shown.

The equation for $\chi_{4}^{\mathrm{B}}$ or rather $d^{4} / d \mu^{4} \Omega$ in terms of partial $\mu$ - and $\Phi_{i}$-derivatives is given in Appendix $\mathrm{D}$ in (D13). Similarily to (35) it can be split in all terms without $L$-derivatives of $\Omega$ and the rest, $\Delta \Omega_{4}$. Apart from the dependence on the two-point correlation functions $\Delta \Omega_{4}$ also carries a dependence of the three- and four point correlation function of the glue potential.

\section{B. Confinement and the hadronic phase}

The analysis of the last section concerning the sensitivity of the fluctuations observables on the details of the
Polyakov loop potential necessitates a detailed discussions of the mechanisms and scales behind the asymptotic behaviour of higher moments in the asymptotic regimes $T / T_{c} \rightarrow 0, \infty$. A particularly interesting regime, however, is the crossover regime at about $T_{c}$, as the fluctuations there may give indirect access to the question of the existence of a critical endpoint in the phase diagram. It is therefore very important to understand, the quantitative knowledge of which couplings is required to make predictions there. It is also of chief interest to pin down the relevant mechanisms in view of the model input, that is not fully controlled. This gives us both, access to the systematic error of the computation as well as the physics in the transition regime.

A particularly helpful observables within this discussion is the kurtosis $\chi_{4}^{\mathrm{B}} / \chi_{2}^{\mathrm{B}}$. For $T / T_{c} \rightarrow 0$ it simply counts the hadronic degrees of freedom. Hence we expect $\chi_{4}^{\mathrm{B}} / \chi_{2}^{\mathrm{B}} \rightarrow 1$. In turn, for $T / T_{c} \rightarrow \infty$ the kurtosis tends towards its perturbative behaviour, $\chi_{4}^{\mathrm{B}} / \chi_{2}^{\mathrm{B}} \rightarrow 2 / 3 \pi^{2}$.

While the high temperature dependence is easily understood in terms of perturbation theory, for $T / T_{c} \rightarrow 0$ the common picture is the following: for these temperatures the Polyakov loop expectation values tend towards zero, $L, \bar{L} \rightarrow 0$. Accordingly, the quark thermal distribution $n_{F}(x, T, L, \bar{L})$ in the presence of the confining backround signaled by $L, \bar{L} \approx 0$ supposedly tends towards a baryonic one. Indeed, taking $L, \bar{L}=0$ in $(26)$ leads us to

$$
\frac{1+2 \bar{L} e^{x / T}+L e^{2 x / T}}{1+3 \bar{L} e^{x / T}+3 L e^{2 x / T}+e^{3 x / T}} \stackrel{L, \bar{L} \rightarrow 0}{\longrightarrow} \frac{1}{1+e^{3 x / T}} .
$$

The right hand side of (37) simply is a baryonic distribution function. Hence, a vanishing Polyakov loop expectation value would effectively lead to baryonic properties. At $\mu=0$ we deduce from (27),

$$
x=\frac{k}{z_{q}} \sqrt{1+\bar{m}_{q, k}^{2}} \gtrsim m_{q, \mathrm{con}} \quad \text { with } \quad m_{q, \mathrm{con}}=\left.\frac{\bar{h} \bar{\sigma}}{2 z_{q}}\right|_{k=0},
$$

is bounded from below by the sum of the temperaturedependent constituent quark mass $m_{q, \text { con }}$ at $k=0$. The $T \rightarrow 0$ limit in 37 then entails, that $e^{3 x / T} \rightarrow \infty$ and $n_{F} \rightarrow e^{-3 x / T}$. The quark part of the grand potential $\Omega$ is proportional to a sum of $n_{F}(x, T, 0,0)+n_{F}(\bar{x}, T, 0,0)$ and the $2 n$ th-order $\mu$-derivatives simply pull down $3^{2 n}$, leading to the desired result of counting baryonic degrees of freedom. Indeed this is seen when directly using $L=$ $\bar{L}=0$ in the model, see Fig. 19 in Appendix D.

Note however, that the above picture involves taking $L, \bar{L} \rightarrow 0$ for $T \rightarrow 0$ and then taking $e^{x / T}$ to $\infty$. A close inspection reveals that this explanation falls short: both limits relate to $T \rightarrow 0$ and the thermal distribution in 37 depends on products of $L, \bar{L}$ and powers of $e^{x / T}$. Indeed, a necessary ingredient for the validity of (37) is

$$
L e^{2 m_{q, \mathrm{con}} / T} \ll 1 .
$$

We shall argue now, that 39 does not hold anywhere, see Fig. 8 for the PQM model under investigation here. 


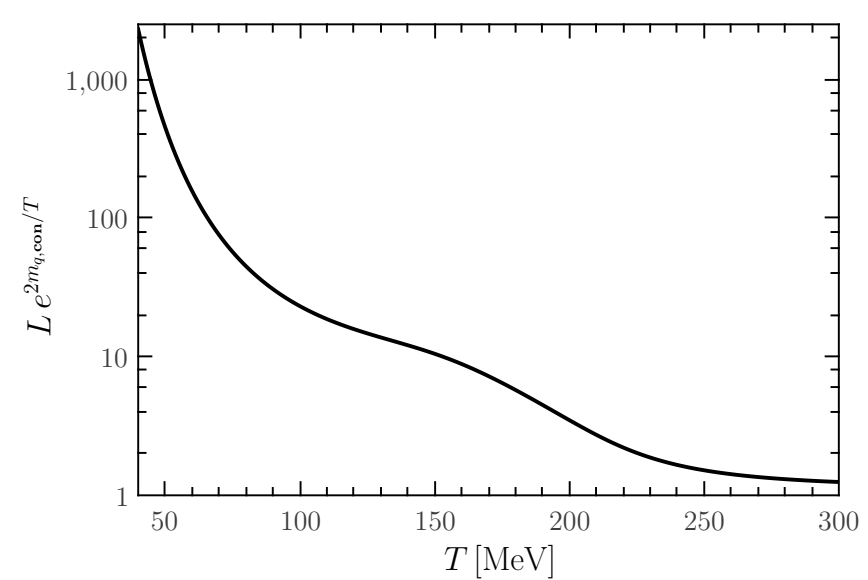

FIG. 8. $L e^{2 m_{q, \text { con }} / T}$ as a function of temperature, obtained in the full computation.

We emphasise that the following argument is done without explicit reference to the specific model and also holds in QCD. To that end we discuss the equations of motion,

$$
\partial_{L} \Omega=\partial_{L} \Omega_{\mathrm{glue}}+\partial_{L} \Omega_{\mathrm{matt}}=0
$$

In a flow equation approach to $\mathrm{QCD}, \Omega_{\text {glue }}$ comprises the contributions from the gluonic and ghost loop in Fig. 1, while $\Omega_{\text {matt }}$ comprises that from the quark and mesonic loop in Fig. 1. The representation in terms of a flow equation facilitates the discussion of the respective scales. Dropping $\Omega_{\text {matt }}$ the grand potential reduces to the pure glue potential with a transition temperature $T_{\text {glue }} \approx 200 \mathrm{MeV}$, see [16, 21, 22]. The glue potential (in the current approximation) features a first order phase transition, and below $T_{\text {glue }}$ the Polyakov loop expectation vanishes. The possible minor center-breaking corrections of the glue propagation due to the vacuum polarisation are neglected. We shall see, that for the present argument we can safely drop these contributions as they only increase the expectation value of the Polyakov loop.

We conclude that the non-vanishing expectation values of $L$ and $\bar{L}$ in QCD are dominantly triggered by the quark loop in $\Omega_{\text {matt }}$. Starting at $T=0$, where the Polyakov loop vanishes, we can estimate its $T$-dependence by computing the thermal derivative. To that end we use the dimensionless potential

$$
V\left[\Phi_{\text {EoM }}\right]=\frac{\Omega}{T^{4}}=V_{\text {glue }}+V_{\text {matt }},
$$

where we have extracted the canonical $T^{4}$ scaling. With (41) the $T$-derivative of 40 normalised with $1 / T^{4}$ reads for small $T / T_{c} \rightarrow 0$

$$
\partial_{T} \partial_{L} V=V_{L L} \partial_{T} L+V_{L \bar{L}} \partial_{T} \bar{L}+V_{L \Phi_{i}} \partial_{T} \Phi_{i}+V_{T L}=0
$$

where $V_{T L}=\left.\partial_{T}\right|_{\Phi} V_{L}$ and index $i$ runs over all fields without $L, \bar{L}$. At $\mu=0$ we have $L=\bar{L}, \partial_{T} L=\partial_{T} \bar{L}$ and we are led to

$$
\partial_{T} L=-\frac{V_{L \Phi_{i}} \partial_{T} \Phi_{i}+V_{T L}}{V_{L L}+V_{L \bar{L}}}
$$

Now we explore the existing knowledge in QCD about the curvature coefficients that contribute to 43 as well as the mixed derivative $V_{T L}$ :

First of all, in the limit $T / T_{c} \rightarrow 0$ the glue potential $V_{\text {glue }, T}$ settles to a finite potential $V_{\text {glue }, T=0}$ with nonvanishing curvature coefficients $V_{L L}, V_{L \bar{L}}, V_{\bar{L} \bar{L}}$, see e.g. 58. Hence the denominator in 43 settles at a finite temperature-independent value. It is left to provide a lower bound for the temperature-dependence of the numerator. The temperature-dependence of the expectation values of $\sigma$ decays at least with the smallest mass scale in QCD, the pion mass. For example, in the PQM model considered in the present work, the only nonvanishing expectation value apart from $L, \bar{L}$ is that of the sigma meson, $\sigma_{0}$. In turn, the mixed field derivatives, e.g. $V_{L \sigma}$ can only couple via the quark sector and hence decay exponentially at least with $e^{-m_{q, \mathrm{con}} / T}$.

It is left to give an estimate for the last term in the numerator, $V_{T L}$. To that end we split the potential $V$ in a center- symmetric part and a center-breaking one. The lowest term in $L, \bar{L}$ in the center-symmetric part is proportional to $L \bar{L}$ and its $L$-derivative is proportional to $\bar{L}$. In turn, the center-breaking terms originate in the quark dynamics and their contributions decay exponentially at least with $e^{-m_{q, \text { con }} / T}$, as is the case in the present model. In QCD the fermionic loop in the model is substituted by a fully interacting one and the constituent quark mass in the decay goes to half of the free energy energy of an interacting quark-anti-quark pair. This leads to the final estimate for the temperature-dependence of $L$ at $\mu=0$,

$$
\partial_{T} L \gtrsim \max \left(c_{\mathrm{q}} e^{-m_{q, \mathrm{con}} / T}, c_{\mathrm{L}} L\right)
$$

with prefactors $c_{1}$ and $c_{\mathrm{L}}$ that carry a potential polynomial dependence on temperature. As the Polyakov loop expectation value is vanishing at $T=0$ we deduce from (44) that

$$
\lim _{T / T_{c} \rightarrow 0} L \rightarrow c(T) e^{-m_{q, \mathrm{con}} / T}
$$

where the prefactor $c(T)$ carries a polynomial temperature dependence. Consequently we do not reach (39) but rather have

$$
L e^{2 x / T} \gg 1,
$$

which concludes our argument. We emphasise again that we have not resorted to any model properties but only have used the scaling properties of the glue and matter parts, $V_{\text {glue }}=\Omega_{\text {glue }} / T^{4}$ and $V_{\text {matt }}=\Omega_{\text {matt }} / T^{4}$ respectively, of the grand potential.

\section{Confinement and baryonic fluctuations}

The analysis in the last section entails the failure of the standard statistical confinement picture based on (37). 


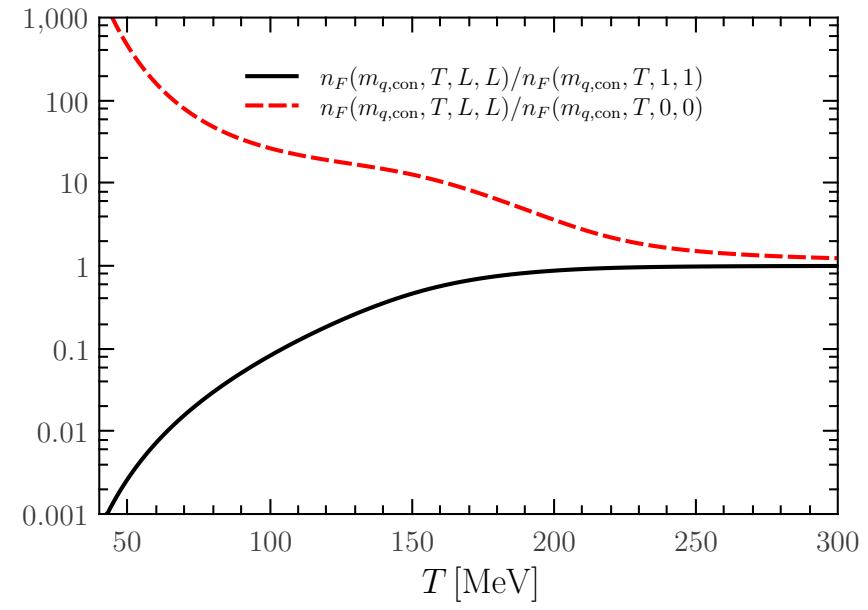

FIG. 9. Ratios of $n_{F}\left(m_{q, \text { con }}, T, L, L\right) / n_{F}\left(m_{q, \text { con }}, T, 0,0\right)$ (full over baryonic distribution) and $n_{F}\left(m_{q, \mathrm{con}}, T, L, L\right) / n_{F}\left(m_{q, \mathrm{con}}, T, 1,1\right) \quad$ (full over quark distribution) as a function of temperature, obtained in the full computation.

Indeed, the latter limit does not hold for any temperature and the Polyakov loop augmented thermal quark distribution never comes even close the baryonic one, see Fig. 9

Instead one can deduce, that the hadronic limit is reached if 46) is satisfied. In the PQM model used here this happens for $T / T_{c} \approx 0.8$, below which we only expect to see the trivial thermal scaling. It is left to show that in this limit we see the hadronic nature of the low temperature phase, that is in particular $\chi_{4}^{\mathrm{B}} / \chi_{2}^{\mathrm{B}} \rightarrow 1$, despite the the thermal distribution for $T / T_{c}$ settling well in between the quark and baryon distribution, see Fig. 9 . Indeed, at $\mu=0$ and $T / T_{c} \rightarrow 0$, and with 45 , the thermal distribution at $k=0$ is well approximated by

$$
n_{F}(x, T, L, \bar{L}) \rightarrow L e^{-x / T} \approx e^{-2 m_{q, \mathrm{con}} / T},
$$

where $x$ in (27), (38) is bounded by $m_{q, \text { con }} / T$ for all $k$. Hence the thermal distribution decays with a thermal correlation length related to the inverse of twice the constituent quark mass. Note also that the quark contribution of (the flow of) the grand potential $\Omega$ is directly proportional to $n_{F}$, see A5 in Appendix A.

With the scaling properties for $T / T_{c} \rightarrow 0$ deduced above we can expand the grand potential in orders of $\exp \left\{-m_{q, \text { con }} / T\right\}$ on the equations of motion. This leaves us with

$$
\begin{aligned}
\Omega= & -\frac{b_{2}}{2} L \bar{L}-\frac{b_{3}}{6}\left(L^{3}+\bar{L}^{3}\right)+\frac{c_{1}}{2}\left[L\left(e^{-x}+2 e^{-2 \bar{x}}\right)\right. \\
& \left.+\bar{L}\left(e^{-\bar{x}}+2 e^{-2 x}\right)\right]+\Omega_{\mathrm{mes}}+O\left(e^{-4 \frac{m_{q, \text { con }}}{T}}\right) .
\end{aligned}
$$

where the $b_{2}, b_{3}$ terms stem from the expansion of the glue potential $\Omega_{\text {glue }}$ in powers of $L, \bar{L}$, see e.g. [53], and we have dropped the subleading term $b_{4} / 4(L \bar{L})^{4}$. The $c_{1}$-term stems from the quark contribution to the grand potential and introduces an explicit breaking of center symmetry. The exponential decay factors such as $e^{-x}$ would carry free energies of half of an interacting quarkanti-quark pair at infinite distance. Here we simply neglect the binding energy and take that of a free quarkanti-quark pair with constituent quark masses as a lower bound. This is also the approximation underlying the current model approximation. As we shall see, the precise values of the parameters $b_{2}, b_{3}, c_{1}$ are irrelevant for the $T \rightarrow 0$ asymptotics of the kurtosis. We also have dropped the mesonic terms as subleading. In (48), $T$ is absorbed into $x$ and $\bar{x}$ for brevity, and $L, \bar{L}$ on the EoM read

$$
\begin{aligned}
& L=\frac{1}{b_{2}}\left[c_{1}\left(e^{-\bar{x}}+2 e^{-2 x}\right)-b_{3} \bar{L}^{2}\right]+O\left(e^{-3 \frac{m_{q, \text { con }}}{T}}\right), \\
& \bar{L}=\frac{1}{b_{2}}\left[c_{1}\left(e^{-x}+2 e^{-2 \bar{x}}\right)-b_{3} L^{2}\right]+O\left(e^{-3 \frac{m_{q, \text { con }}}{T}}\right) .
\end{aligned}
$$

Eq. 49 makes explicit the expansion in powers of $\exp \left\{-m_{q, \text { con }} / T\right\}$. All terms dropped in (48) are at least of the order $\exp \left\{-4 m_{q, \text { con }} / T\right\}$. It also supports the nice heuristic interpretation of the Polyakov loop expectation values $L, \bar{L}$ being related to the free energy of a test quark/anti-quark: the leading order of $L, \bar{L}$ decay exponentially with $\exp \left\{-\left(m_{q, \text { con }} \pm \mu\right) / T\right\}$ respectively. The implicit $\bar{L}^{2}$ and $L^{2}$ terms encode terms of at least quadratic order in $\exp \left\{-\left(m_{q, \text { con }} \pm \mu\right) / T\right\}$. Eq. 49 also entails that at finite chemical potential $\bar{L}$ is enhanced relative to $L$. Inserting the respective equations for $L, \bar{L}$ on the right hand sides of 49 leads us to

$$
\begin{aligned}
& L=\frac{c_{1}}{b_{2}} e^{-\bar{x}}+\left(2 \frac{c_{1}}{b_{2}}-b_{3} \frac{c_{1}^{2}}{b_{2}^{3}}\right) e^{-2 x}+O\left(e^{-3 \frac{m_{q, \text { con }}}{T}}\right), \\
& \bar{L}=\frac{c_{1}}{b_{2}} e^{-x}+\left(2 \frac{c_{1}}{b_{2}}-b_{3} \frac{c_{1}^{2}}{b_{2}^{3}}\right) e^{-2 \bar{x}}+O\left(e^{-3 \frac{m_{q, \text { con }}}{T}}\right) .
\end{aligned}
$$

Eq. (50) reveals the composite nature of the Polyakov loop expectation values. For example, $L$ has an expansion in quarks and anti-diquarks all of which carry the same $S U(N)$ representation. Hence we expect $L \bar{L}$ to carry both mesonic $(q \bar{q})$ and baryonic properties $(q q q$, $\bar{q} \bar{q} \bar{q})$. Indeed, it follows from (50) that

$$
\begin{aligned}
L \bar{L}= & \frac{c_{1}^{2}}{b_{2}^{2}} e^{-2 \frac{m_{q, \text { con }}}{T}} \\
& +\left(2 \frac{c_{1}^{2}}{b_{2}^{2}}-b_{3} \frac{c_{1}^{3}}{b_{2}^{4}}\right)\left[e^{-3 x}+e^{-3 \bar{x}}\right]+O\left(e^{-4 \frac{m_{q, \text { con }}}{T}}\right) .
\end{aligned}
$$

The first term in 51) carries no baryon number and heuristically relates to mesons, while the second one carries baryon number, there are no diquark contributions. The occurance of the decay with multiples of the constituent quark mass $e^{-2 x}$ for $q \bar{q}$ and $e^{-3 x}$ for $q q q$ relates 
to the fact that the Polyakov loop terms describe pairs and triplets of quarks rather than the bound states.

In case of the baryons this is related to the missing higher order quark interactions in the current model that carry the interaction energy of the hadronic states. This can be amended by taking into account multi-quark interactions with or without dynamical hadronisation.

In case of the mesons the missing contributions are hidden in the mesonic part of the grand potential. To see this more clearly we proceed with the expansion of the full grand potential. Note first that $\Omega$ in 48 only has linear and bilinear terms in $L$ and $\bar{L}$ evaluated on their EoMs, (49), as well as cubic terms. The latter cubic terms carry baryon number in leading order. Using $\left(L \partial_{L}+\bar{L} \partial_{\bar{L}}\right) \Omega=0$ to express the linear terms with the bilinear and cubic ones we arrive at

$$
\begin{aligned}
\Omega & =\frac{b_{2}}{2} \bar{L} L+\frac{b_{3}}{3}\left(L^{3}+\bar{L}^{3}\right)+\Omega_{\mathrm{mes}}+O\left(e^{-4 \frac{m_{q, \mathrm{con}}}{T}}\right) \\
& =\Omega_{q q q}+\Omega_{q \bar{q}}+\Omega_{\mathrm{mes}}
\end{aligned}
$$

with

$$
\begin{aligned}
& \Omega_{q q q}=\left(\frac{c_{1}^{2}}{b_{2}}-\frac{b_{3}}{6} \frac{c_{1}^{3}}{b_{2}^{3}}\right)\left(e^{-3 x}+e^{-3 \bar{x}}\right)+O\left(e^{-4 \frac{m_{q, \text { con }}}{T}}\right) \\
& \Omega_{q \bar{q}}=\frac{c_{1}^{2}}{2 b_{2}} e^{-2 \frac{m_{q, \text { con }}}{T}}+O\left(e^{-4 \frac{m_{q, \text { con }}}{T}}\right)
\end{aligned}
$$

Note that the EoM also imply that the linear terms have the form in (51) with additional contributions in the first line in (53) steming from the $L^{3}+\bar{L}^{3}$-term. With 52 the grand potential takes its final baryonic-type form. It has a $\mu$-independent offset and subleading contributions. The $\Omega_{q q q}$ term carries baryon number with contributions from all three terms in the grand potential. In terms of the Polyakov loop it has the form $L^{3}+\bar{L}^{3}$. The mechanism unraveled above only holds for $L \bar{L} \rightarrow 0$ which signals the hadronic phase.

Note also that the total mesonic contribution is given by $\Omega_{q \bar{q}}+\Omega_{\text {mes }}$. The latter part dominates in the hadronic phase and shows the correct mesonic mass scales. We expect a similar phenomenon for the baryonic part once the baryonic states are included in the effective action. Note however, that the baryons do not affect the dynamics of the theory due to their heavy masses which suppressed any baryonic off-shell fluctuations at all momentum scales.

Now we are in the position to discuss the fate of the kurtosis deep in the hadronic regime, that is for low temperatures $T / T_{c} \ll 1$. From 52 we deduce, that even powers of total $\mu$-derivatives of the grand potential are simply proportional to the potential in the $T \rightarrow 0$ limit,

$$
\frac{d^{2 n} \Omega}{d(\mu / T)^{2 n}}=3^{2 n} \Omega_{q q q}+O\left(e^{-4 \frac{m_{q, \mathrm{con}}}{T}}\right),
$$

with $\Omega_{q q q}$ counts three-quark states, the effective

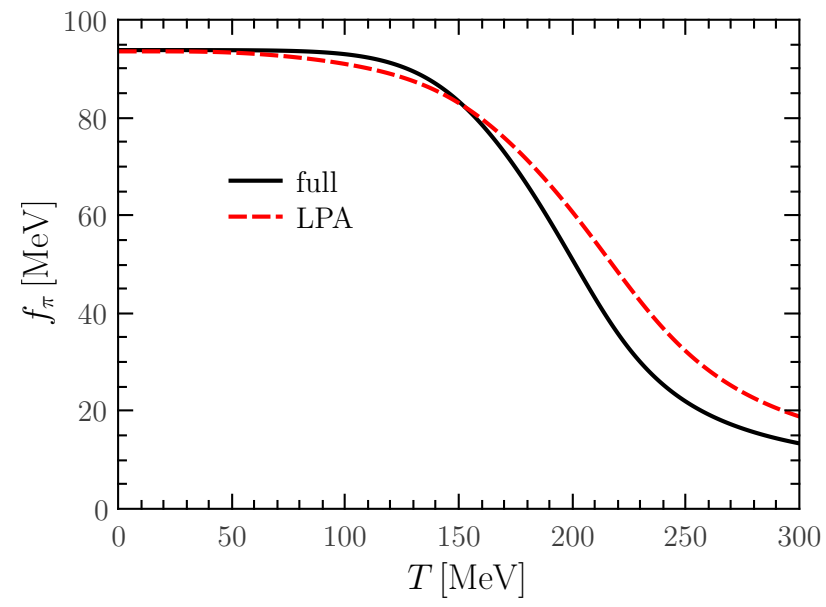

FIG. 10. Pion decay constant as a function of $T$, calculated in the PQM with LPA and full approximation.

baryons. Eq. (54) implies in particular

$$
\frac{\chi_{4}^{\mathrm{B}}}{\chi_{2}^{\mathrm{B}}}=1+O\left(e^{-\frac{m_{q, \mathrm{con}}}{T}}\right),
$$

In summary, despite the final outcome being the same, the mechanism behind the asymptotic $T$-scaling of the kurtosis and also the higher moments is different to that suggested in the literature: in contradistinction to former claims the asymptotic low energy scaling is obtained in a regime where the thermal distribution are far from being baryonic, see Figs. 89. Indeed, the Polyakov loop enhanced thermal distribution is far from the baryonic one for any temperature, as is clear from the above analysis.

\section{Results}

In the last section we have reached a better understanding of the mechnisms taking place in the hadronic low energy/low temperature regime of QCD. Most of the properties discussed do indeed not depend on the model under investigation but are properties of QCD. With this in mind we finally come to the numerical computations of this paper for the QCD-enhanced $N_{f}=2 \mathrm{PQM}$ model. Before we present the results, let us briefly summarise the approximations used here: We build upon the approximation tested in Section III for the quark-meson model. We use a full effective meson potential $V_{k}(\phi ;, L, \bar{L})$ that includes multi-meson scatterings. The dependence on the Polyakov loop variables $L, \bar{L}$ is implicit as the flow is evaluated on given backgrounds $L, \bar{L}$. We also introduce scale-dependent wave function renormalisations $Z_{\phi}(L, \bar{L}), Z_{q}(L, \bar{L})$ with the same implicit dependence on $L, \bar{L}$. The $Z$ 's account for the change of the dispersions for both mesons and quarks. Additionally we allow for scale-dependent quark-meson interaction $h_{k}(L, \bar{L})$. The quark-meson part in the $L, \bar{L}$ background is amended with a QCD-enhanced Polyakov loop potential $V_{\text {glue }}(L, \bar{L})$ 

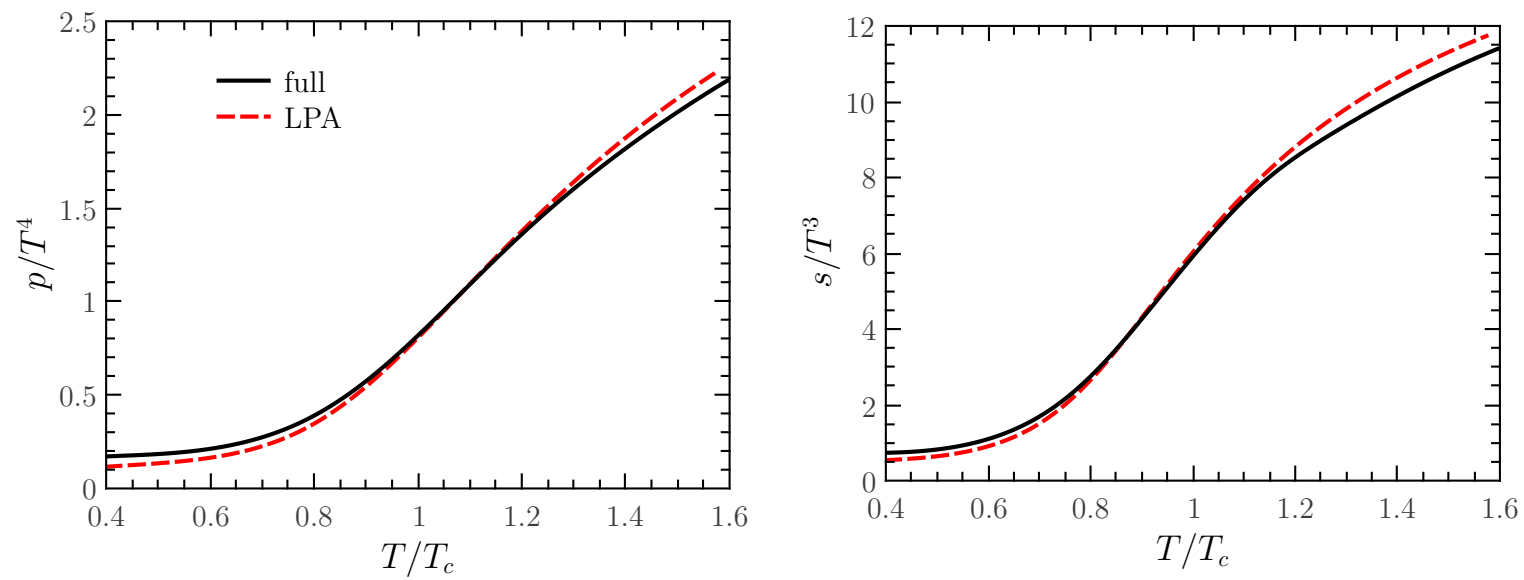

FIG. 11. Pressure (left panel) and entropy density (right panel) as functions of $T / T_{c}$. The psudo-critical temperature is determined by the peak of $\left|\partial \bar{\rho}_{0, k=0} / \partial T\right|, T_{c}=181 \mathrm{MeV}, 190 \mathrm{MeV}$ for full and LPA, respectively.
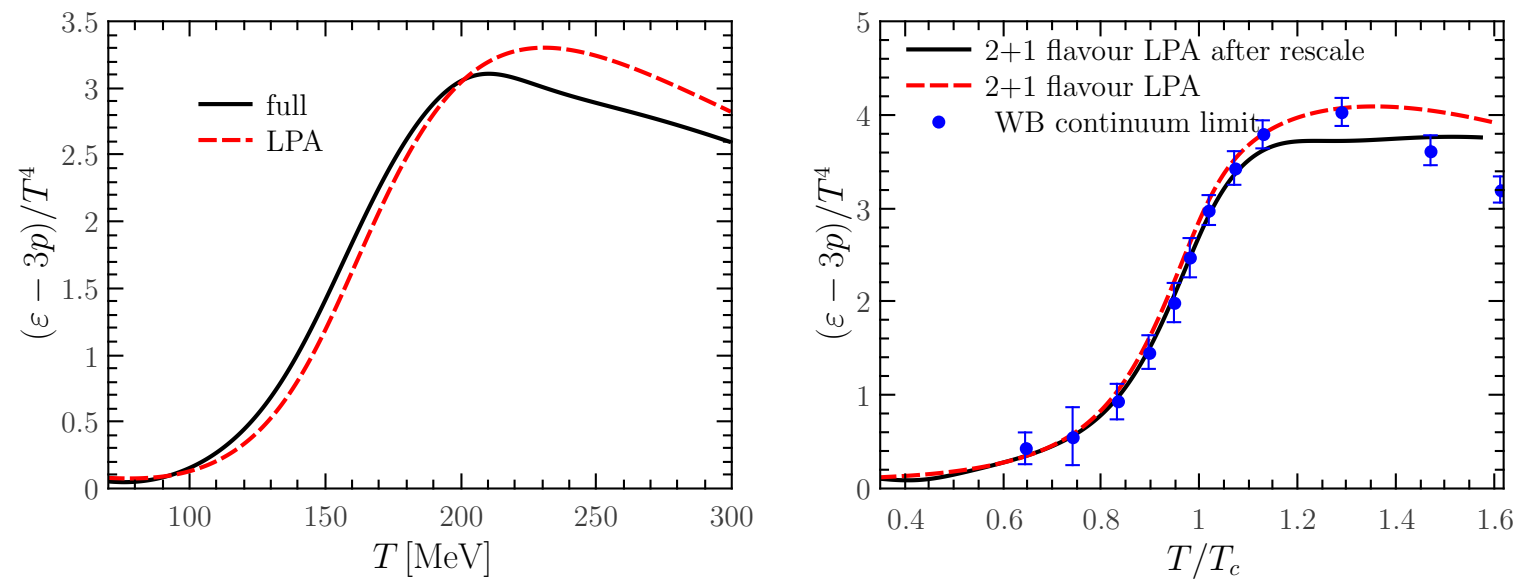

FIG. 12. Left panel: trace anomaly as a function of $T$. Right panel: trace anomaly for $N_{f}=2+1$ in LPA from 22 (red dashed line), multiplied by the ratio of the full $N_{f}=2$ data over the LPA data (black solid line), in comparison to lattice result from Wuppertal-Budapest collaboration [66].

in Eq. (32). The potential is a standard model potential with the correct temperature scaling of QCD, see [16, 21, 22]. Our full results are compared with results in the standard LPA approximation as has been used in 22 for $N_{f}=2$ and $N_{f}=2+1$ flavour QCD.

In Fig. 10 we show the pion decay constant as a function of temperature. As in the QM model the additional fluctuations from the wave function renormalisation and the Yukawa coupling lead to a sharper chiral crossover. In 22] it has been shown that in the $2+1$ flavour case there is a very good agreement of both pressure and trace anomaly as functions of reduced temperature for $T / T_{c} \lesssim 1.2$ with the corresponding lattice results of the Wuppertal Budapest group, see Figure 5 there. Note that the pressure even agrees well above this scale. However, the trace anomaly, which is more sensitive to the correct counting of fluctuations, shows a widening gap. The LPA results overshoot the lattice results which hints a both, an incorrect counting of fluctuations at large scale as well as the impact of the ultraviolet cutoff.
As already mentioned before, the agreement between the $2+1$ and 2 flavour results as functions of reduced temperature is still remarkable for $T / T_{c} \lesssim 1$. This supports the point of view that the dominant effect of the strange quark on the thermodynamics at temperatures $T \lesssim T_{c}$ is to change the overall scale. For larger momentum or temperature scales, however, its dynamics contributes inevitably. Keeping this in mind we compare the LPA and full pressure and entropy in Fig. 11 as functions of reduced temperature. The additional fluctuations introduces via the wave function renormalisations and the Yukawa couplings have little impact on the thermodynamics. We expect this property to hold also for $2+1$ flavours. Hence the already very good agreement of the pressure and entropy in LPA is not spoiled.

The trace anomaly, left panel of Fig. 12, also does not change significantly for temperatures below $T_{c}$. Above $T_{c}$, the full results undershoots the LPA result. Again we expect the $2+1$ result to show the same change. However, in this regime we are sensitive to cutoff effects 

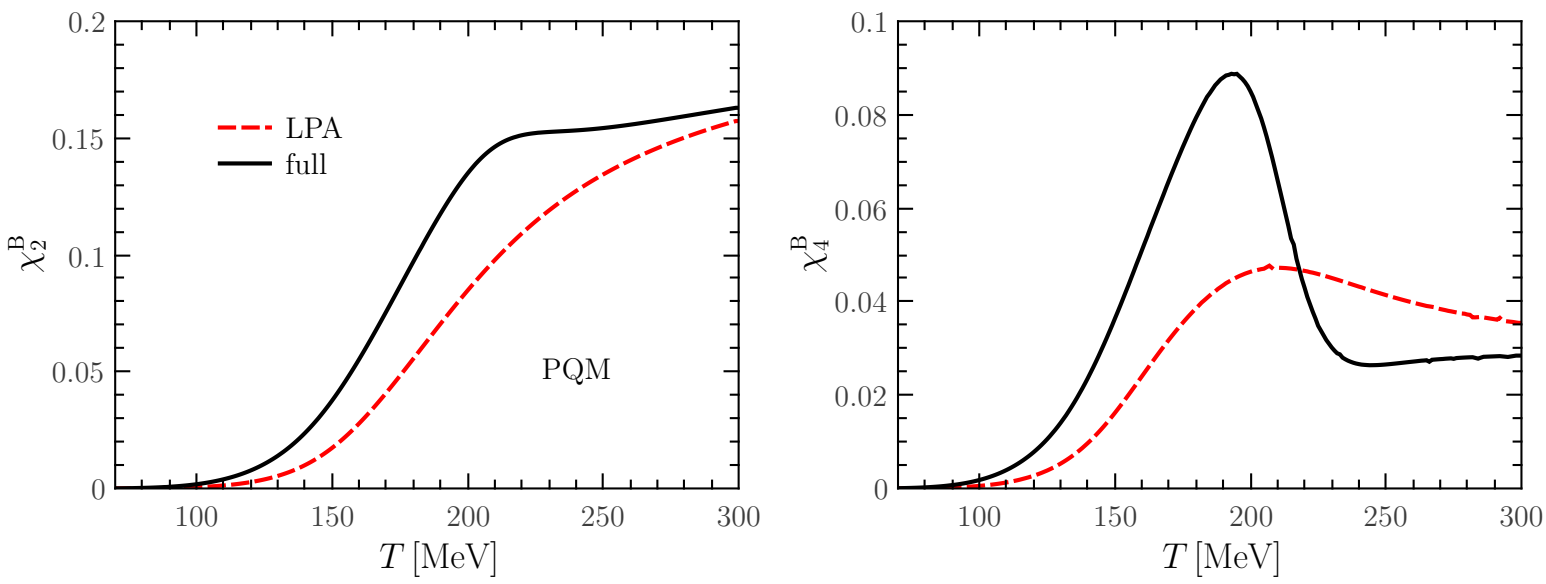

FIG. 13. Quadratic (left panel) and quartic (right panel) baryon number fluctuations as functions of $T$ in the PQM model in LPA and full truncation.

and missing fluctuations in the first place.

We conclude that the additional quantum and thermal fluctuations considered in the present work have very little impact on the thermodynamics below $T_{c}$, despite their significant change of the matter fluctuations as shown in the QM model. Above $T_{c}$ they have a larger impact as can be seen from the trace anomaly, left panel of Fig. 12 . In a bold attempt to estimate the results of a $2+1$-flavour computation we also show the $N_{f}=2+1$ data in LPA for the trace anomaly from 22], multiplied by the ratio of the full $N_{f}=2$ data over the LPA data, see right panel of Fig. 12. As expected, the quantitative agreement with the lattice data is not spoiled for $T \lesssim T_{c}$. For temperatures $T \gtrsim T_{c}$ one clearly sees the effects of the ultraviolet cutoff and the missing fluctuations.

Finally we come to the baryonic fluctuations. The numerical results for the second and fourth moment are shown in Figure 13. We emphasise that the computation of the $\mu$-derivatives of the grand potential at small temperatures requires the careful evaluation of the genuine thermal and chemical potential scaling of quark anomalous dimension and Yukawa coupling, for more details see Appendix C. Interestingly, the difference of the full results to that in LPA is significant in clear contradistinction to the situation for the thermodynamics. This is expected as the higher moments by definition carry the details of the fluctuation physics in the model or theory at hand. From Fig. 13 one observes once more that the quadratic and quartic baryon number fluctuations obtained with the full approximation, are larger and change more rapidly during the chiral crossover. Note that larger fluctuations are important in view of experimental measurements, whose statistical error increase significantly for higher fluctuations.

In Fig. 14 we present the final results on the kurtosis. As expected, the significant change from the LPA results to the result of the full approximation propagates from that in the second and fourth moments $\chi_{2}^{\mathrm{B}}, \chi_{4}^{\mathrm{B}}$. This is good news in terms of experimental measurements, and provides a very good test for the convergence of the present approximation scheme towards the full result. Such an analysis also requires a discussion of the systematic error. The grey band in Fig. 14 provides a rough estimate of the systematic error of the full computation arising from the UV-cutoff scale $\Lambda$, through the following simple formula [22]:

$$
\frac{\chi_{4}^{\mathrm{B}}}{\chi_{2}^{\mathrm{B}}} \pm \Delta \frac{\chi_{4}^{\mathrm{B}}}{\chi_{2}^{\mathrm{B}}}=\frac{\chi_{4}^{\mathrm{B}}}{\chi_{2}^{\mathrm{B}}}\left(1 \pm \frac{4}{e^{\Lambda / T}-1}\right)
$$

with $\Lambda=700 \mathrm{MeV}$. Note that this error estimate does not include the effects of the correct hadronic decoupling above $T_{c}$ which in the present framework is most easily implemented with dynamical hadronisation. Moreover, at even higher scales gluonic fluctuations have to be added. Finally, for temperatures $T \lesssim 0.8 T_{c}$ we expect frequency-dependences in particular of the fermionic couplings to further improve the reliability of the results. This estimate is based on a computation of fermionic couplings one the first fermionic Matsubara frequency $\pi T$ and the improved one used in the present work, that takes into account the correct thermal and $\mu$-scaling. The latter computation implements effectively the effects of full frequency dependences, for details see the Appendix C. The results start to disagree for $T \lesssim 0.8 T_{c}$ and we expect a compution with full frequency-dependences to decrease the systematic error further in this regime. A more detailed analysis in an upgraded approximation will be discussed elsewhere.

The expected asymptotic value 1 for the kurtosis is obtained at low temperature in both LPA and the full approximation. We also compare the kurtosis of baryon multiplicity distribution with that of the continuumextrapolated lattice result from Wuppertal-Budapest collaboration [6]. The latter is a $2+1$ quark flavour result with physical quark masses. In the spirit of the previous discussion about the dominant strange quark effect as a different total scale setting in $2+1$ flavour QCD in comparison to 2 flavour QCD we rescale the temperature 

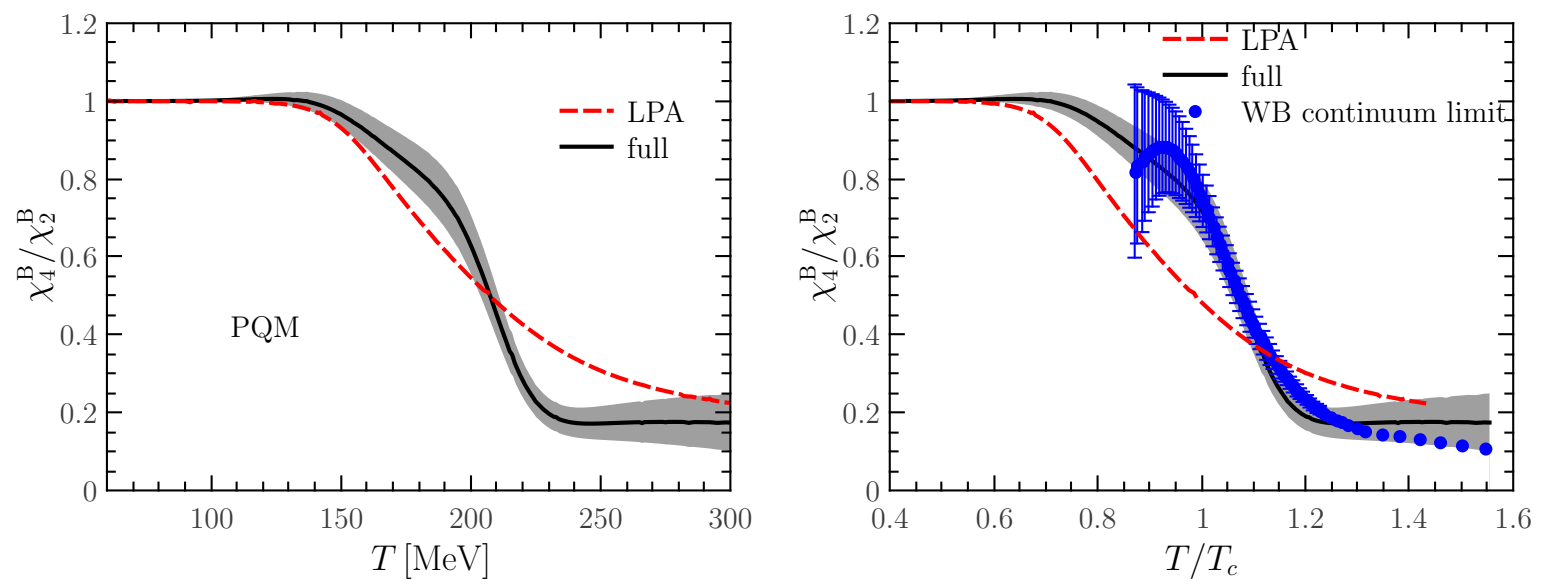

FIG. 14. Left panel: Kurtosis of baryon number distribution in the PQM model. The grey band shows an error estimate for the full computation, resulting from the UV-cutoff scale. Right panel: Comparison with the continuum-extrapolated lattice results from Wuppertal-Budapest collaboration [6]. The temperature is rescaled by their corresponding pseudo-critical temperature $T_{c}$.

in units of the pseudo-critical temperature $T_{c}$. For the definition of the absolut temperature scale we choose the peak position in the fourth moment $\chi_{4}^{\mathrm{B}}$, as this is easily accessible in both approaches. From the right panel of Fig. 13, one can easily read $T_{c}=193 \mathrm{MeV}, 210 \mathrm{MeV}$ for full and LPA, respectively. For the $2+1$ flavor lattice simulation, $T_{c}=155 \mathrm{MeV}$ is obtained from the results of $\chi_{4}^{\mathrm{B}}[8$, which is also consistent with the calculations in [67. With such a normalisation one finds that the full calculation is in very good agreement with the lattice result for temperature $T \lesssim T_{c}$, while the LPA is not, see Fig. 14. The most significant difference is the rapid convergence of the kurtosis in the full computation and the lattice result towards 1 . This entails that the dominance of the hadronic nature for $T \leq T_{c}$ is approached very quickly, which also has an impact on the rapid chemical freeze-out that is observed experimentally. The present approach allows to study the relative importance of glue, quark, mesonic and baryonic flcutuations which will provide further insights in this intriguing question.

\section{SUMMARY AND OUTLOOK}

In this work we have studied the phase structure, thermodynamics, and in particular higher moments of baryon multiplicity distribution, in low energy effective models with matter and glue fluctuations. The fluctuations have been taken into account with the functional renormalisation group. This has allowed us to investigate in detail the impact of the matter and glue dynamics on baryon number fluctuations and the kurtosis of baryon number distribution.

Furthermore, we have provided new insights on the mechanism of quark confinement in terms of effective degrees of freedom: the common picture in low energy effective models is that effective quark confinement is realized through an effective baryonic thermal distributions, called statistical confinement. A detailed study unravels that the effective thermal distribution is quite far from the baryonic one, and the deviation grows exponentially when $T / T_{c}$ approaches zero. Instead of an effective baryonic thermal distribution we find that the center symmetry of the glue potential plays a key role, which guarantees that the degree of freedom is baryonic at low temperature.

For a detailed study of the rôle of fluctuations several different approximations to the full low energy effective theory of 2 flavour QCD have been employed. These extended approximations have been compared with the standard LPA truncation, that allows for a very efficient inclusion of meson fluctuations and has been considered in the literature. We find that the non-trivial frequency and momentum dependence of the propagators strenghens the temperature-driven chiral crossover. In another words, the non-trivial dispersion introduced by quantum and thermal fluctuations increases the transition strength. This effect is observed in all observables considered in this work: the order parameter of phase transition, the pressure, the entropy, trace anomaly and baryonic fluctuation observables $\chi_{2}^{\mathrm{B}}$ and $\chi_{4}^{\mathrm{B}}$. Interestingly, while the effects on order parameter and thermodynamical observables is very small, it is significant for the higher moments. This is very well compatible with the fact, that the results for $2+1$ QCD for order parameter and thermodynamics including the trace anamaly were already in quantitative agreement with the lattice results for $T \lesssim T_{c}$ for the LPA approximation.

We have also provided estimates for the trace anomaly, see Fig. 12 and the kurtosis, see Fig. 14 in $2+1$ flavour QCD. This is based on the comparison of 2 flavour with $2+1$ flavour computations in 22 as well as $2+1$ with $2+1+1$ flavour computations in 54. This suggests that the predominant effect of strange and charm quark is 
the simple rescaling of the total momentum scale which is done by presenting the results as functions of the reduced temperature. In the validity regime of the present truncation to low energy QCD, for temperatures $T \lesssim T_{c}$, the present results agree very well with the corresponding lattice results. This provides further support for the reliability and the convergence of the present approximation scheme of the QCD-enhanced model for these temperature regime.

Finally we discuss our findings in view of the experiments: the present analysis predicts larger baryonic fluctuations in QCD at finite density in comparison to model results in the literature obtained in LPA. This effect is quite prominent and, thus, it may play an important role in the observed kurtosis of the net-proton multiplicity distributions at RHIC.

Technically, it has been shown in the literature that meson fluctuations beyond the mean-field approximation smoothen the chiral crossover. This also leads to a significantly reduced critical region in comparison to mean field results as well as small baryonic fluctuations. In summary the mesonic fluctuation analysis made the search for a critical end point far more difficult. The present analysis emphasises the relevance of fermionic fluctuations. The latter naturally strengthen the crossover and in particular enhance baryonic fluctuations observables. This increases both, the likelihood of a critical endpoint as well as its observability via fluctuation measurements. This is in line with the observation that the measurements of higher moments of the net-proton distributions in the first phase of BES program at RHIC show that their energy dependence can neither be reproduced by transport models without critical point contributions, nor by a hadron resonance gas model 68. Hence, although more analyses and explanations are needed to clarify the measurements, the QCD critical point is still a fascinating candidate.

The calculations in this work have been performed in a QCD-enhanced low energy effective model. Its limitations at $T \gtrsim T_{c}$ as well as the missing frequencydependence of the couplings have been discussed in detail. Ongoing extensions of the current work concern $2+1$ flavour QCD, the frequency-dependence relevant in particular at small temperatures $T \lesssim 0.8 T_{c}$, the missing glue fluctuations and hadronic decoupling at $T \gtrsim T_{c}$. The latter is considered within dynamical hadronisation. These improvements entail a full embedding of the present low energy effective theory in continuum QCD in the FRG framework as done in the fQCD collaboration [69. We hope to report on related results in near future.

\section{ACKNOWLEDGMENTS}

We thank T. K. Herbst, M. Mitter, F. Rennecke, B.-J. Schaefer and N. Strodthoff for valuable discussions and work on related subjects. W.-J. Fu thanks also the support of Alexander von Humboldt Foundation. This work is supported by the Helmholtz Alliance HA216/EMMI, and by ERC-AdG-290623.

\section{Appendix A: Regulators and threshold functions}

In the present work we use $3 d$ flat regulators, 46, 47, for quarks and mesons. They read

$$
\begin{aligned}
& R_{k}^{\phi}\left(q_{0}, \vec{q}\right)=Z_{\phi, k} \vec{q}^{2} r_{B}\left(\vec{q}^{2} / k^{2}\right), \\
& R_{k}^{q}\left(q_{0}, \vec{q}\right)=Z_{q, k} i \vec{\gamma} \cdot \vec{q} r_{F}\left(\vec{q}^{2} / k^{2}\right),
\end{aligned}
$$

with

$$
\begin{aligned}
& r_{B}(x)=\left(\frac{1}{x}-1\right) \Theta(1-x), \\
& r_{F}(x)=\left(\frac{1}{\sqrt{x}}-1\right) \Theta(1-x) .
\end{aligned}
$$

In order to simplify our expressions for the threshold functions, we define

$$
\begin{aligned}
G_{\phi}\left(q, \bar{m}_{\phi, k}^{2}\right) & =\frac{1}{z_{\phi} \tilde{q}_{0}^{2}+1+\bar{m}_{\phi, k}^{2}}, \\
G_{q}\left(q, \bar{m}_{q, k}^{2}\right) & =\frac{1}{z_{q}^{2}\left(\tilde{q}_{0}+i \tilde{\mu}\right)^{2}+1+\bar{m}_{q, k}^{2}},
\end{aligned}
$$

with $\tilde{q}_{0}=q_{0} / k, \tilde{\mu}=\mu / k$, and $q_{0}=\left(2 n_{q}+1\right) \pi T\left(n_{q} \in\right.$ $\mathbb{Z}$ ) for fermions and $2 n_{q} \pi T$ for bosons. Note that $z=$ $Z^{\|} / Z^{\perp}$, which are the ratios between the longitudinal and transverse wave function renormalisation factors, are chosen to be 1 throughout this paper. First of all, we define

$$
\begin{aligned}
\mathcal{F}_{(1)}\left(\bar{m}_{q, k}^{2}, z_{q} ; T, \mu\right) & =\frac{T}{k} \sum_{n_{q}} G_{q}\left(q, \bar{m}_{q, k}^{2}\right), \\
\mathcal{B}_{(1)}\left(\bar{m}_{\phi, k}^{2}, z_{\phi} ; T,\right) & =\frac{T}{k} \sum_{n_{q}} G_{\phi}\left(q, \bar{m}_{\phi, k}^{2}\right) .
\end{aligned}
$$

Summing up the Matsubara frequencies yields

$$
\begin{aligned}
& \mathcal{F}_{(1)}\left(\bar{m}_{q, k}^{2}, z_{q} ; T, \mu\right)=\frac{1}{2 z_{q} \sqrt{1+\bar{m}_{q, k}^{2}}} \\
& \quad \times\left(1-n_{F}\left(\bar{m}_{q, k}^{2}, z_{q} ; T, \mu\right)-n_{F}\left(\bar{m}_{q, k}^{2}, z_{q} ; T,-\mu\right)\right),
\end{aligned}
$$

and

$$
\begin{aligned}
\mathcal{B}_{(1)} & \left(\bar{m}_{\phi, k}^{2}, z_{\phi} ; T\right) \\
= & \frac{1}{z_{\phi}^{1 / 2} \sqrt{1+\bar{m}_{\phi, k}^{2}}}\left(\frac{1}{2}+n_{B}\left(\bar{m}_{\phi, k}^{2}, z_{\phi} ; T\right)\right),
\end{aligned}
$$


with the distribution functions

$$
\begin{aligned}
& n_{B}\left(\bar{m}_{\phi, k}^{2}, z_{\phi} ; T\right) \\
= & \frac{1}{\exp \left\{\frac{1}{T} \frac{k}{z_{\phi}^{1 / 2}}\left(1+\bar{m}_{\phi, k}^{2}\right)^{1 / 2}\right\}-1},
\end{aligned}
$$

and

$$
\begin{aligned}
& n_{F}\left(\bar{m}_{q, k}^{2}, z_{q} ; T, \mu\right) \\
= & \frac{1}{\exp \left\{\frac{1}{T}\left[\frac{k}{z_{q}}\left(1+\bar{m}_{q, k}^{2}\right)^{1 / 2}-\mu\right]\right\}+1} .
\end{aligned}
$$

The threshold functions appearing in the flow equation for the effective potential, i.e. Eq. (8), are given by

$$
\begin{aligned}
& l_{0}^{(B, d)}\left(\bar{m}_{\phi, k}^{2}, \eta_{\phi, k} ; T\right) \\
= & \frac{2}{d-1}\left(1-\frac{\eta_{\phi, k}}{d+1}\right) \mathcal{B}_{(1)}\left(\bar{m}_{\phi, k}^{2}, z_{\phi}=1 ; T\right),
\end{aligned}
$$

and

$$
\begin{aligned}
& l_{0}^{(F, d)}\left(\bar{m}_{q, k}^{2}, \eta_{q, k} ; T, \mu\right) \\
= & \frac{2}{d-1}\left(1-\frac{\eta_{q, k}}{d}\right) \mathcal{F}_{(1)}\left(\bar{m}_{q, k}^{2}, z_{q}=1 ; T, \mu\right) .
\end{aligned}
$$

Furthermore, we also need

$$
\mathcal{F}_{(n)}\left(\bar{m}_{q, k}^{2}, z_{q} ; T, \mu\right)=\frac{T}{k} \sum_{n_{q}}\left(G_{q}\left(q, \bar{m}_{q, k}^{2}\right)\right)^{n}
$$

which are easily obtained from $\mathcal{F}_{(1)}$ through

$$
\mathcal{F}_{(n+1)}\left(\bar{m}_{q, k}^{2}, z_{q} ; T, \mu\right)=-\frac{1}{n} \frac{\partial}{\partial \bar{m}_{q, k}^{2}} \mathcal{F}_{(n)}\left(\bar{m}_{q, k}^{2}, z_{q} ; T, \mu\right)
$$

The threshold function $\mathcal{B B}_{(2,2)}$ is derived from

$$
\mathcal{B B}_{(2,2)}\left(\bar{m}_{\phi_{a}, k}^{2}, \bar{m}_{\phi_{b}, k}^{2}, z_{\phi} ; T\right)
$$

$$
=\frac{\partial^{2}}{\partial \bar{m}_{\phi_{a}, k}^{2} \partial \bar{m}_{\phi_{b}, k}^{2}} \mathcal{B} \mathcal{B}_{(1,1)}\left(\bar{m}_{\phi_{a}, k}^{2}, \bar{m}_{\phi_{b}, k}^{2}, z_{\phi} ; T\right),
$$

with

$$
\begin{aligned}
& \mathcal{B B}_{(1,1)}\left(\bar{m}_{\phi_{a}, k}^{2}, \bar{m}_{\phi_{b}, k}^{2}, z_{\phi} ; T\right) \\
= & \frac{T}{k} \sum_{n_{q}} G_{\phi}\left(q, \bar{m}_{\phi_{a}, k}^{2}\right) G_{\phi}\left(q, \bar{m}_{\phi_{b}, k}^{2}\right) .
\end{aligned}
$$

Its analytic expression, after the Matsubara frequencies are summed up, is given by

$$
\begin{aligned}
& \mathcal{B B}_{(1,1)}\left(\bar{m}_{\phi_{a}, k}^{2}, \bar{m}_{\phi_{b}, k}^{2}, z_{\phi} ; T\right) \\
= & -\frac{1}{z_{\phi}^{1 / 2}}\left\{\left(\frac{1}{2}+n_{B}\left(\bar{m}_{\phi_{a}, k}^{2}, z_{\phi} ; T\right)\right) \frac{1}{\left(1+\bar{m}_{\phi_{a}, k}^{2}\right)^{1 / 2}}\right. \\
& \times \frac{1}{\left(\bar{m}_{\phi_{a}, k}^{2}-\bar{m}_{\phi_{b}, k}^{2}\right)}+\left(\frac{1}{2}+n_{B}\left(\bar{m}_{\phi_{b}, k}^{2}, z_{\phi} ; T\right)\right) \\
& \left.\times \frac{1}{\left(1+\bar{m}_{\phi_{b}, k}^{2}\right)^{1 / 2}} \frac{1}{\left(\bar{m}_{\phi_{b}, k}^{2}-\bar{m}_{\phi_{a}, k}^{2}\right)}\right\}
\end{aligned}
$$

In the same way, by means of derivatives with respect to appropriate masses, threshold functions $\mathcal{F B}$ 's in Eq. (C4) are easily obtained with

$$
\begin{aligned}
& F B_{(1,1)}\left(\bar{m}_{q, k}^{2}, \bar{m}_{\phi, k}^{2}, z_{q}, z_{\phi} ; T, \mu, p_{0}\right)=\frac{T}{k} \sum_{n_{q}} G_{\phi}\left(p-q, \bar{m}_{\phi, k}^{2}\right) G_{q}\left(q, \bar{m}_{q, k}^{2}\right) \\
= & \frac{1}{2} \frac{k^{2}}{z_{\phi} z_{q}^{2}}\left\{-n_{B}\left(\bar{m}_{\phi, k}^{2}, z_{\phi} ; T\right) \frac{z_{\phi}^{1 / 2}}{\left(1+\bar{m}_{\phi, k}^{2}\right)^{1 / 2}} \frac{1}{\left(i p_{0}-\mu+\frac{k}{z_{\phi}^{1 / 2}}\left(1+\bar{m}_{\phi, k}^{2}\right)^{1 / 2}\right)^{2}-\left(1+\bar{m}_{q, k}^{2}\right)\left(\frac{k}{z_{q}}\right)^{2}}\right. \\
& -\left(n_{B}\left(\bar{m}_{\phi, k}^{2}, z_{\phi} ; T\right)+1\right) \frac{z_{\phi}^{1 / 2}}{\left(1+\bar{m}_{\phi, k}^{2}\right)^{1 / 2}} \frac{1}{\left(i p_{0}-\mu-\frac{k}{z_{\phi}^{1 / 2}}\left(1+\bar{m}_{\phi, k}^{2}\right)^{1 / 2}\right)^{2}-\left(1+\bar{m}_{q, k}^{2}\right)\left(\frac{k}{z_{q}}\right)^{2}} \\
& +n_{F}\left(\bar{m}_{q, k}^{2}, z_{q} ; T,-\mu\right) \frac{1}{\left(1+\bar{m}_{q, k}^{2}\right)^{1 / 2}} \frac{z_{q}}{\left(i p_{0}-\mu-\frac{k}{z_{q}}\left(1+\bar{m}_{q, k}^{2}\right)^{1 / 2}\right)^{2}-\left(1+\bar{m}_{\phi, k}^{2}\right) \frac{k^{2}}{z_{\phi}}} \\
& \left.+\left(n_{F}\left(\bar{m}_{q, k}^{2}, z_{q} ; T, \mu\right)-1\right) \frac{1}{\left(1+\bar{m}_{q, k}^{2}\right)^{1 / 2}} \frac{\left(i p_{0}-\mu+\frac{k}{z_{q}}\left(1+\bar{m}_{q, k}^{2}\right)^{1 / 2}\right)^{2}-\left(1+\bar{m}_{\phi, k}^{2}\right) \frac{k^{2}}{z_{\phi}}}{\left({ }_{p}\right.}\right\} .
\end{aligned}
$$


The $T \rightarrow 0$ limit of A16 is achieved by simply dropping the bosonic thermal distribution functions, $n_{B} \rightarrow 0$, as well as reducing the fermionic ones to Heaviside theta functions, $n_{F}\left(\bar{m}_{q, k}^{2}, z_{q} ; T, \pm \mu\right) \rightarrow \theta\left(k / z_{q}\left(1+\bar{m}_{q, k}^{2}\right)^{1 / 2} \mp \mu\right)$. The latter limit signals the silver blaze property: below the critical chemical potential $\mu=\mu_{*}$ no observable shows a dependence on the chemical potential. Note that for $T \rightarrow 0$ there is still a $\mu$-dependence on $p_{0}-i \mu$. Again this relates to the silver blaze property as observables relate to $p_{0}$-integrals, and the $\mu$-dependence can be removed by a simple shift of the integration contour below the first pole in the complex plane at $\mu=\mu_{*}$. Consequently, on the level of the threshold functi $F B_{(1,1)}$ in A16 there is a qualitative difference between the $\mu$ dependence in the quark distribution functions $n_{F}$ and that on the frequency $p_{0}+i \mu$ : only upon frequency integration both $\mu$-dependences show the characteristic exponential dependence on $\exp \{ \pm \mu / T\}$ relevant for the silver blaze property. This is investigated in detail in Appendix C.

Here we proceed with the relevant threshold functions

$$
\begin{aligned}
& F B_{(2,1)}\left(\bar{m}_{q, k}^{2}, \bar{m}_{\phi, k}^{2}, z_{q}, z_{\phi} ; T, \mu, p_{0}\right) \\
= & \frac{T}{k} \sum_{n_{q}} G_{\phi}\left(p-q, \bar{m}_{\phi, k}^{2}\right)\left(G_{q}\left(q, \bar{m}_{q, k}^{2}\right)\right)^{2} \\
= & -\frac{\partial}{\partial \bar{m}_{q, k}^{2}} F B_{(1,1)}\left(\bar{m}_{q, k}^{2}, \bar{m}_{\phi, k}^{2}, z_{q}, z_{\phi} ; T, \mu, p_{0}\right),
\end{aligned}
$$

and

$$
\begin{aligned}
& F B_{(1,2)}\left(\bar{m}_{q, k}^{2}, \bar{m}_{\phi, k}^{2}, z_{q}, z_{\phi} ; T, \mu, p_{0}\right) \\
= & \frac{T}{k} \sum_{n_{q}}\left(G_{\phi}\left(p-q, \bar{m}_{\phi, k}^{2}\right)\right)^{2} G_{q}\left(q, \bar{m}_{q, k}^{2}\right) \\
= & -\frac{\partial}{\partial \bar{m}_{\phi, k}^{2}} F B_{(1,1)}\left(\bar{m}_{q, k}^{2}, \bar{m}_{\phi, k}^{2}, z_{q}, z_{\phi} ; T, \mu, p_{0}\right) .
\end{aligned}
$$

Note that the functions $F B_{(1,1)}, F B_{(2,1)}$, and $F B_{(1,2)}$ are complex valued at non-zero chemical potential and, thus, we have to project them onto the real axis, as explained in the text, viz.

$$
\mathcal{F} \mathcal{B}_{(n, m)}=\operatorname{Re}\left(F B_{(n, m)}\right),
$$

with $z_{\phi}=z_{q}=1$. The threshold function in the flow of the Yukawa coupling (C5) can be expressed as

$$
\begin{aligned}
& L_{(1,1)}^{(d)}\left(\bar{m}_{q, k}^{2}, \bar{m}_{\phi, k}^{2}, \eta_{q, k}, \eta_{\phi, k} ; T, \mu\right) \\
= & \frac{2}{d-1}\left[\left(1-\frac{\eta_{\phi, k}}{d+1}\right) \mathcal{F} \mathcal{B}_{(1,2)}\left(\bar{m}_{q, k}^{2}, \bar{m}_{\phi, k}^{2} ; T, \mu, p_{0, \mathrm{ex}}\right)\right. \\
& \left.+\left(1-\frac{\eta_{q, k}}{d}\right) \mathcal{F} \mathcal{B}_{(2,1)}\left(\bar{m}_{q, k}^{2}, \bar{m}_{\phi, k}^{2} ; T, \mu\right)\right],
\end{aligned}
$$

with the fixed frequency $p_{0, \text { ex }}$ to be specified in Appendix $\mathrm{C}$.

\section{Appendix B: Anomalous dimensions of the mesons}

We have discussed the flow equation for the zero-point correlation function, i.e. the effective potential, in Section III A. In order to investigate more interesting properties embedded in the meson and quark propagators, one has to go beyond this order. Differentiating both sides of Eq. (6) with respect to fields twice, one arrives at the flow equations for two-point functions. After performing appropriate projects, one can obtain the desired flow equations. For example, the meson anomalous dimension can be obtained with

$$
\eta_{\phi, k}\left(p_{0}, \vec{p}\right)=-\left.\frac{1}{Z_{\phi, k}} \frac{1}{N_{f}^{2}-1} \frac{\partial^{2}}{\partial|\vec{p}|^{2}} \frac{\delta^{2} \partial_{t} \Gamma_{k}}{\delta \pi_{i}(-p) \delta \pi_{i}(p)}\right|_{\rho=\kappa},
$$

where a sum over the $N_{f}^{2}-1$ pions is implied. The flow is evaluated at the expansion point $\kappa$ of the Taylor expansion in the mesonic field, as the right hand sides of the flows are evaluated at this point. Note that another projection procedure for $\eta$ relates to finite differences, e.g. $\partial_{t} \Gamma_{k}^{(2)}\left(p_{0}=0, \vec{p}^{2}=k^{2}\right)-\partial_{t} \Gamma_{k}^{(2)}(0)$, as has been used in [70, 71]. This gives better access to the global change of the wave function in the relevant momentum regime $\vec{p}^{2} \leq k^{2}$. However, it has been shown in 24 that a Taylor expansion about $q=0$ works quantitatively (for a mesonic $O(N)$-model) for momenta $q^{2} \lesssim k^{2}$. This origintaes in the infrared regularisation of these momenta. As $\eta_{\phi, k}$ is only inserted in the right hand side of flows with loop momenta $\vec{q}^{2} \leq k^{2}$ we use $\vec{q}^{2}=0$ as the evaluation momentum. We also choose vanishing frequency $q_{0}=0$ in order to keep an expansion point that has the underlying Euclidean $O(4)$-symmetry. Note, however, that the regulator used breaks the $O(4)$-symmetry and we will reevaluate this choice in the case of the fermionic anomalous dimension.

In summary this leads us to

$$
\begin{aligned}
\eta_{\phi, k}= & \eta_{\phi, k}(0,0) \\
= & \frac{1}{6 \pi^{2}}\left\{\frac{4}{k^{2}} \bar{\kappa}_{k}\left(\bar{V}_{k}^{\prime \prime}\left(\bar{\kappa}_{k}\right)\right)^{2} \mathcal{B} \mathcal{B}_{(2,2)}\left(\bar{m}_{\pi, k}^{2}, \bar{m}_{\sigma, k}^{2} ; T\right)\right. \\
& +N_{c} \bar{h}_{k}^{2}\left[\left(2 \eta_{q, k}-3\right) \mathcal{F}_{(2)}\left(\bar{m}_{q, k}^{2} ; T, \mu\right)\right. \\
& \left.\left.-4\left(\eta_{q, k}-2\right) \mathcal{F}_{(3)}\left(\bar{m}_{q, k}^{2} ; T, \mu\right)\right]\right\}
\end{aligned}
$$

where the threshold functions $\mathcal{B B}_{(2,2)}$ and $\mathcal{F}_{(n)}$ are defined in [23], and are presented in Appendix A] as well.

\section{Appendix C: Flows of the fermionic couplings}

In the spirit of the derivative expansion used for the present class of models the flow of the fermionic couplings is obtained by evaluating the corresponding derivatives w.r.t. the fields at low frequencies and momenta. These 
flows are given by

$$
\begin{aligned}
& \eta_{q, k}\left(p_{0}, \vec{p}\right)=\frac{1}{Z_{q, k}\left(p_{0}, \vec{p}\right)} \frac{1}{4 N_{c} N_{f}} \\
& \times \operatorname{Re}\left[\left.\frac{\partial^{2}}{\partial|\vec{p}|^{2}} \operatorname{Tr}\left(i \vec{\gamma} \cdot \vec{p}\left(-\frac{\delta^{2} \partial_{t} \Gamma_{k}}{\delta \bar{q}(-p) \delta q(p)}\right)\right)\right|_{\rho=\kappa}\right],
\end{aligned}
$$

for the anomalous dimension and

$$
\begin{aligned}
& \partial_{t} h_{k}\left(p_{0}, \vec{p}\right)=\frac{\sqrt{2 N_{f}}}{\sigma} \frac{1}{4 N_{c} N_{f}} \\
& \times \operatorname{Re}\left[\left.\operatorname{Tr}\left(-\frac{\delta^{2} \partial_{t} \Gamma_{k}}{\delta \bar{q}(-p) \delta q(p)}\right)\right|_{\rho=\kappa}\right],
\end{aligned}
$$

for the Yukawa coupling. The results in 24 for the simple spatial momentum dependence for $\vec{q}^{2} \lesssim k^{2}$ of the mesons carries over to the quarks as it originates in the infrared regularisation of these momenta. Hence we use $\vec{q}=0$.

It is left to specify the frequency at which (C1) and C2 are evaluated. We have already discussed briefly below A16 in Appendix A that the $\mu$-dependence in the argument $p_{0}+i \mu$ only reflects the correct physical behavior if keeping the full frequency dependence. Hence, within an evaluation of the fermionic flows at a fixed frequency we face subtleties that relate to the silver blaze problem: all couplings with fermionic legs have an explicit $T$ and $\mu$-dependence as well as one in the frequency argument. At finite temperature and chemical potential the latter is a sum of the fermionic matsubara frequency and the chemical potential, $p_{0}+i \mu=2 \pi T(n+1 / 2)+i \mu$ with $n \in \mathbb{Z}$. The former explicit dependence shows the standard thermal suppression as well as that of the parameters such as the masses and couplings. This is clearly seen in (A16) where the explicit $\mu$ and $T$-dependence is that of the fermionic distribution functions $n_{F}$ as well as that of $\bar{m}_{\phi / q}^{2}, z_{\phi / q}$.

We conclude that inserting $p_{0}+i \mu$-dependent anomalous dimensions in the loops leads to standard thermal sums, and finally to additional thermal factors $n_{F}$ and $1+n_{F}$ related to thermal distribution functions as in (A16). Related results will be considered elsewhere.

In turn, frequency-independent wave function renormalisations are obtained by evaluating $\eta_{q, k}\left(p_{0}+i \mu, \vec{p}\right)$ at some fixed small fermionic Matsubara frequency $p_{0}=$ $2 \pi T(n+1 / 2)$. This introduces an artificial temperaturedependence. Note also that this subtlety is not related to the specific regulator used but rather applies to all regulators. For small cutoff scales $k \lesssim T$ this is a subleading effects, for $k \gg T$, however, thermal effects should be suppressed exponentially with $\exp \left\{-c\left(R_{k}\right) k / T\right\}$ with a cutoff dependent coefficient $c\left(R_{k}\right)$, see 72]. For the present 3d flat cutoff we have $c\left(R_{k}\right)=1 / 2$ leading to the standard thermal distribution functions already for the anomalous dimensions.

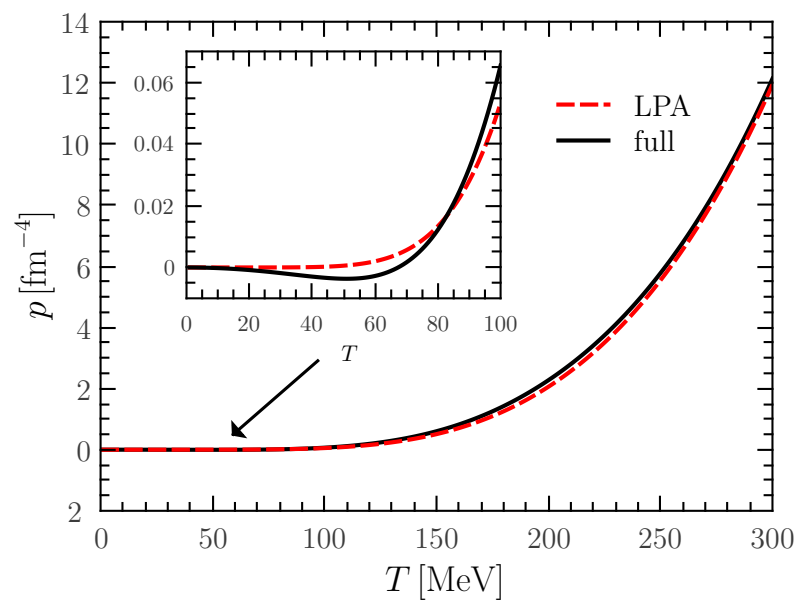

FIG. 15. Pressure in unit of $\mathrm{fm}^{-4}$ as a function of $T$ with truncations full and LPA. The inset plot zooms in to the region $T=0 \sim 100 \mathrm{MeV}$.

The articficial temperature-dependence is sub-leading as it is polynomially suppressed with powers of $T / k$ but it may play a qualitative rôle in temperature regimes where the observables at hand is small. Moreover, the larger the canonical momentum dimension is, the bigger is the effect. In Fig. 15 we show the pressure of the QM model in the low temperature regime. There, it is exponentially suppressed with $\exp \left\{-m_{\pi} / T\right\}$ and hence the above cutoff effects play a rôle. Here, $\eta_{q, k}$ and $h_{k}$ are obtained by evaluating the corresponding flows, $(\mathrm{C} 1)$ and $(\mathrm{C} 2 \mathrm{sim}$ ply on the first Matsubara frequency $\pi T$. The discussion above explains the negative pressure seen in Fig. 15 for the QM-model in the full approximation.

Evidently, this problem is resolved by improving the approximation to frequency-dependent quark anomalous dimensions $\eta_{q}(p)$ and Yukawa couplings $h(p)$. Here we resort to an effective resolution that does not force us to crank up the approximation: on the right hand side of the flows both $\eta_{q}$ and $h$ are evaluated for momenta $\vec{q}^{2} \leq k^{2}$. The integrands decay proportional to $\vec{q}^{2}$ for small momenta $\vec{q}^{2} / k^{2} \rightarrow 0$ and are maximal for momenta $\vec{q}^{2} / k^{2} \approx 1$. This anyway suggests an evaluation of the flows for $\eta_{q}$ and $h$ at $\vec{q}^{2} \approx k^{2}$. The present approximation assumes $O(4)$-symmetry of the effective action also at finite $k$, and hence the couplings are assumed to be functions of $q_{0}^{2}+\vec{q}^{2}$. Thus, we will evaluate the flows (C1) and (C2) at $p_{0}=k$ for $k \gtrsim \pi T$. This resolves the problem of the artificial temperature dependence.

For $k \lesssim \pi T$ the minimal momentum squared is bounded by the lowest Matsubara frequency and hence $p^{2} \geq(\pi T)^{2}$. This suggests an evaluation at

$$
p_{0, \mathrm{ex}}^{2}=k^{2}+(\pi T)^{2} \theta_{T}(k / T),
$$

with some smoothened version $\theta_{T}$ of the heavyside function with $\theta_{T}(k / T \ll 1) \rightarrow 1$ and $\theta(k / T \gg 1) \rightarrow 0$ reflecting the known thermal decay, e.g. $\theta_{T}(x)=\exp \{-2 x\}$. Such a $\theta_{T}(2 k / T)$ overestimates the effect of the Matsubara sum and we take the conservative choice $\exp \{-2 / 5 x\}$ 

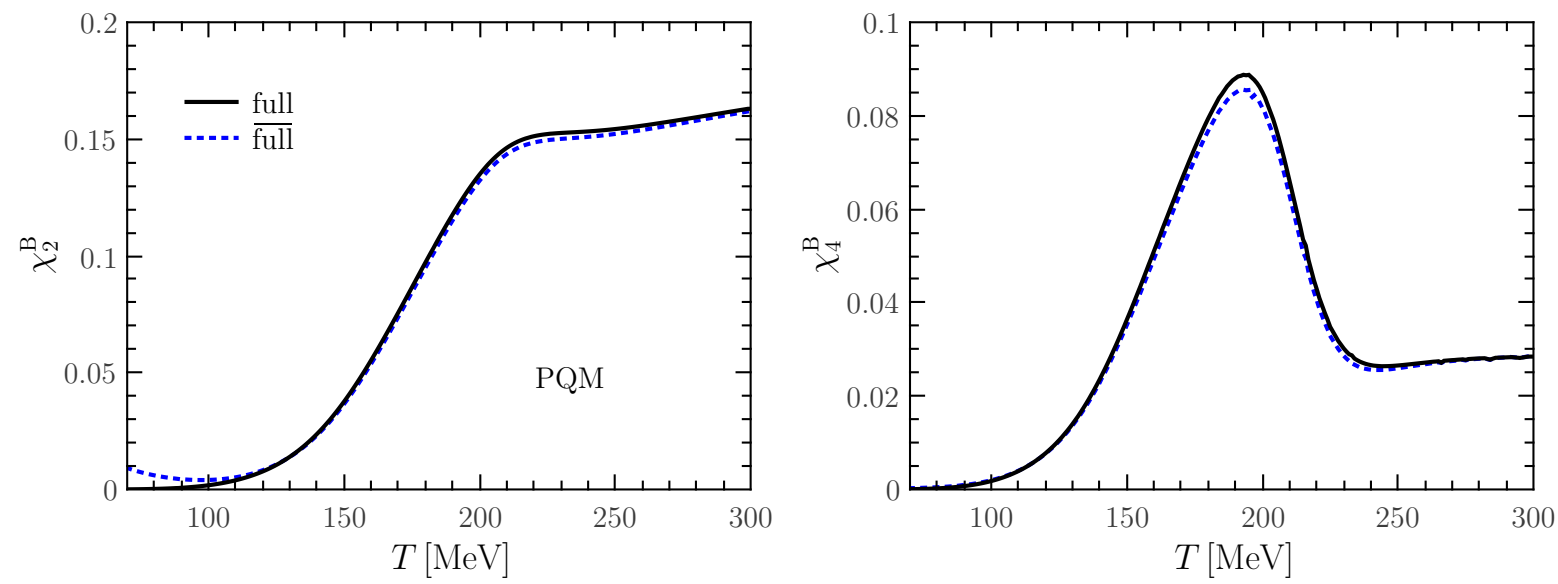

FIG. 16. Quadratic (left panel) and quartic (right panel) baryon number fluctuations in the full approximation with external frequency $p_{0}+i \mu=p_{0, \mathrm{ex}}$, denoted by full, and that with external frequencies $p_{0}+i \mu=\pi T+i \mu$, denoted by full.

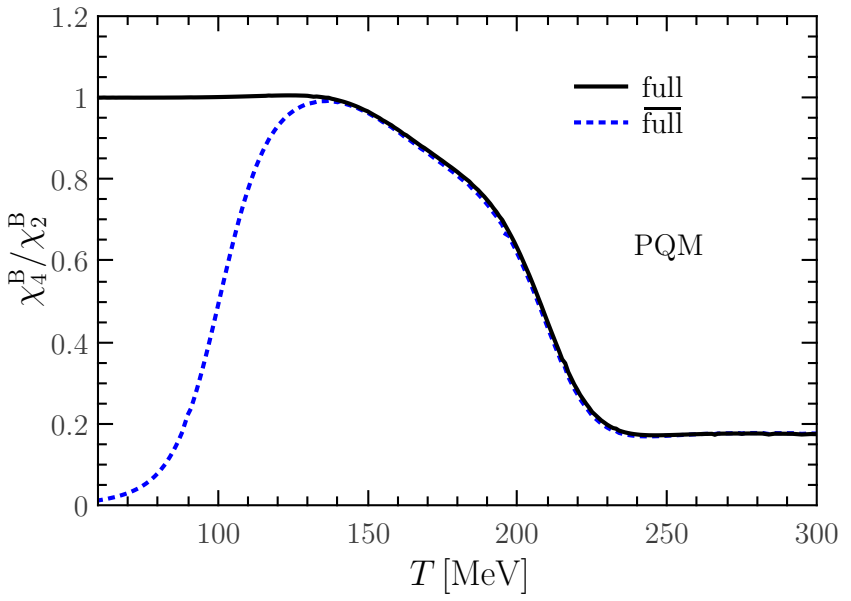

FIG. 17. Same as Fig. 16, but for the kurtosis of baryon number distribution.

which guarantees the decoupling for large cutoff scales $k / T \gg 1$ and does not influence the thermal behaviour for $k / T \lesssim 1$.

The above discussion of the temperature-dependence applies as well to the dependence on the chemical potential. An evaluation of $\eta_{q}$ at a fixed Matsubara-frequency destroys the relation between $\mu$ and $p_{0}$-derivatives and induces an artificial $\mu$-dependencies. Again this may play a rôle for small temperatures where $\mu$-derivaties tend towards zero. Hence, in the present work we simply drop the sub-leading $\mu$-dependencies and set $p_{0}+i \mu \rightarrow p_{0}$ in A16 and the following equations. In summary we arrive at the anomalous dimension

$$
\begin{aligned}
\eta_{q, k}= & \frac{1}{24 \pi^{2} N_{f}}\left(4-\eta_{\phi, k}\right) \bar{h}_{k}^{2} \\
& \times\left\{\left(N_{f}^{2}-1\right) \mathcal{F} \mathcal{B}_{(1,2)}\left(\bar{m}_{q, k}^{2}, \bar{m}_{\pi, k}^{2} ; T, \mu, p_{0, \mathrm{ex}}\right)\right. \\
& \left.+\mathcal{F} \mathcal{B}_{(1,2)}\left(\bar{m}_{q, k}^{2}, \bar{m}_{\sigma, k}^{2} ; T, \mu,, p_{0, \mathrm{ex}}\right)\right\}
\end{aligned}
$$

and the flow of the Yukawa coupling

$$
\begin{aligned}
\partial_{t} \bar{h}_{k}= & \left(\frac{\eta_{\phi, k}}{2}+\eta_{q, k}\right) \bar{h}_{k}+\frac{1}{4 \pi^{2} N_{f}} \bar{h}_{k}^{3} \\
\times & \left\{-\left(N_{f}^{2}-1\right) L_{(1,1)}^{(4)}\left(\bar{m}_{q, k}^{2}, \bar{m}_{\pi, k}^{2}, \eta_{q, k}, \eta_{\phi, k} ; T, \mu, p_{0, \mathrm{ex}}\right)\right. \\
& \left.+L_{(1,1)}^{(4)}\left(\bar{m}_{q, k}^{2}, \bar{m}_{\sigma, k}^{2}, \eta_{q, k}, \eta_{\phi, k} ; T, \mu, p_{0, \mathrm{ex}}\right)\right\}
\end{aligned}
$$

Let us now compare the results for $\chi_{2}^{\mathrm{B}}, \chi_{4}^{\mathrm{B}}$ and the kurtosis $\chi_{2}^{\mathrm{B}} / \chi_{2}^{\mathrm{B}}$ for the full aproximation with consistent $T$ and $\mu$-dependence leading to (C4), (C5) with the computation with fixed frequency $p_{0}+i \mu=\pi T+i \mu$, denoted by full. The results for the second and fourth moment $\chi_{2}^{\mathrm{B}}, \chi_{4}^{\mathrm{B}}$ and for the kurtosis $\chi_{2}^{\mathrm{B}}, \chi_{4}^{\mathrm{B}}$ are presented in Figs. 16 17. Evidently, both approximations are consistent with each other except for low temperatures, as can be seen in particular for the second moment in Fig. 16. These deviations are even more obvious in the kurtosis, Fig. 17. where the deviation below $T \approx 150 \mathrm{MeV}$ is significant, that is for $T \lesssim 0.8 T_{c}$. The full apropximation, expanded at $p_{0}+i \mu \rightarrow p_{0, \text { ex }}$ has a $T, \mu$-consistent scaling and exhibits the correct asymptotic behavior of the kurtosis for low temperature in Eq. (55). In turn, an expansion at $p_{0}+i \mu \rightarrow p_{0, \mathrm{ex}}+i \mu$ fails for temperatures $T \lesssim 0.8 T_{c}$ 


\section{Appendix D: Baryon number fluctuations and vertex expansions}

The generalised suceptibilities in (21) involve total $\mathrm{nth}$ $\mu_{B}$-derivatives of the pressure $p=-\Omega\left[\Phi_{\mathrm{EoM}}\right]$, where $\Phi_{\mathrm{EoM}}$ are the solutions of the equations of motion of all fields in the model, $\Phi=(L, \bar{L}, q, \bar{q}, \sigma, \vec{\pi})$. These derivatives hit the explicit $\mu_{B}$-dependence of the grand potential, and its implicit ones in the couplings as well as that of $\Phi_{\mathrm{EoM}}$.

In the following we discuss $\mu=\mu_{B} / 3$-derivatives of the grand potential $\Omega$. For example, its first $\mu$-derivative reads

$$
\frac{d \Omega\left[\Phi_{\mathrm{EoM}}\right]}{d \mu}=\left.\partial_{\mu}\right|_{\Phi} \Omega+\partial_{\mu} \Phi_{i, \mathrm{EoM}} \frac{\partial \Omega}{\partial \Phi_{i}}=\partial_{\mu} \Omega,
$$

where the evaluation of the right hand side at $\Phi=\Phi_{\text {EoM }}$ is understood. In (D1) we have used the equations of motion, and the partial derivatives are at fixed $\Phi$. In (D1) and the following relation we shall use, that the total $\mu$-derivative can be split in a partial $\mu$-derivative at fixed field and a part that hits the implicit $\mu$-dependence of $\Phi_{\mathrm{EoM}}$, to wit

$$
\frac{d}{d \mu}=\partial_{\mu}+\partial_{\mu} \Phi_{i, \mathrm{EoM}} \frac{\partial}{\partial \Phi_{i}},
$$

With (D2) the second $\mu$-derivative follows as

$$
\frac{d^{2} \Omega\left[\Phi_{\mathrm{EoM}}\right]}{d \mu^{2}}=\frac{d}{d \mu} \partial_{\mu} \Omega=\partial_{\mu}^{2} \Omega+\partial_{\mu} \Phi_{i, \mathrm{EoM}} \frac{\partial^{2} \Omega}{\partial \Phi_{i} \partial \mu} .
$$

Eq. D3 depends on the $\partial_{\mu} \Phi_{i, \text { EoM }}$. This can be computed from the $\mu$-derivatives of the respective EoM, to wit

$$
\frac{d}{d \mu} \frac{\partial \Omega}{\partial \Phi_{i}}=\frac{\partial^{2} \Omega}{\partial \mu \partial \Phi_{i}}+\partial_{\mu} \Phi_{j, \mathrm{EoM}} \Omega_{i j}^{(2)}=0,
$$

with

$$
\Omega_{i j}^{(2)}=\frac{\partial^{2} \Omega}{\partial \Phi_{i} \partial \Phi_{j}}
$$

This can be resolved for $\partial_{\mu} \Phi_{\mathrm{EoM}}$ leading to

$$
\partial_{\mu} \Phi_{i, \mathrm{EoM}}=-G_{i j} \Omega_{\mu j}
$$

where, for the sake of brevity, we have introduced the notation

$$
\Omega_{\mu^{n} i_{1} \ldots i_{m}}=\frac{\partial^{n+m} \Omega}{\partial \mu^{n} \partial \phi_{i_{1}} \cdots \partial \phi_{i_{m}}} \quad \text { and } \quad G_{i j}=\left[\frac{1}{\Omega^{(2)}}\right]_{i j},
$$

which also keeps the higher $\mu$-derivatives simple. Inserting (D6) in (D3) and using the notation (D7) finally leads to

$$
\frac{d^{2} \Omega\left[\Phi_{\mathrm{EoM}}\right]}{d \mu^{2}}=\Omega_{\mu^{2}}-\Omega_{\mu i} G_{i j} \Omega_{\mu j}
$$

Now we proceed to the third $\mu$-derivative of $\Omega$. Within our short hand notation (D7), and using (D6), the total $\mu$-derivative, (D2), takes the form

$$
\frac{d}{d \mu}=\partial_{\mu}-\Omega_{\mu i} G_{i j} \frac{\delta}{\delta \Phi_{j}} .
$$

Applying (D9) to (D8) leads us to

$$
\frac{d^{3} \Omega\left[\Phi_{\mathrm{EoM}}\right]}{d \mu^{3}}=\Omega_{\mu^{3}}-\Omega_{\mu i} G_{i j} \Omega_{\mu^{2} j}-\frac{d}{d \mu}\left[\Omega_{\mu i} G_{i j} \Omega_{\mu j}\right] .
$$

The total $\mu$-derivative in the second term on the right hand side can also be performed with

$$
\begin{aligned}
\frac{d}{d \mu} \Omega_{\mu^{n} i_{1} \ldots i_{m}} & =\Omega_{\mu^{n+1} i_{1} \ldots i_{m}}-\Omega_{\mu^{n} i_{1} \ldots i_{m} k} G_{k s} \Omega_{\mu s} \\
\frac{d}{d \mu} G_{i j} & =-G_{i k}\left(\Omega_{\mu k l}-\Omega_{k l r} G_{r s} \Omega_{\mu s}\right) G_{l j}
\end{aligned}
$$

following from (D9). Then we finally arrive at

$$
\begin{aligned}
\frac{d^{3} \Omega\left[\Phi_{\mathrm{EoM}}\right]}{d \mu^{3}}= & \Omega_{\mu^{3}}-3 \Omega_{\mu j} G_{j i} \Omega_{\mu^{2} i}+3 \Omega_{\mu l} G_{l k} \Omega_{\mu j} G_{j i} \Omega_{\mu k i} \\
& -\Omega_{\mu i} G_{i r} \Omega_{\mu l} G_{l k} \Omega_{\mu j} G_{j s} \Omega_{k r s} . \quad(\mathrm{D} 12)
\end{aligned}
$$

For the kurtosis we need the fourth $\mu$-derivative. It can be straightforwardly derived from (D12) with (D9) and (D11), and leads us to

$$
\begin{aligned}
& \frac{d^{4} \Omega\left[\Phi_{\mathrm{EoM}}\right]}{d \mu^{4}} \\
= & \Omega_{\mu^{4}}-4 \Omega_{\mu j} G_{j i} \Omega_{\mu^{3} i}-3 \Omega_{\mu^{2} i} G_{i j} \Omega_{\mu^{2} j} \\
& +12 \Omega_{\mu^{2} l} G_{l k} \Omega_{\mu j} G_{j i} \Omega_{\mu k i}+6 \Omega_{\mu l} G_{l k} \Omega_{\mu j} G_{j i} \Omega_{\mu^{2} k i} \\
& -6 \Omega_{\mu l} G_{l k} \Omega_{\mu j} G_{j r} \Omega_{\mu^{2} i} G_{i s} \Omega_{k r s} \\
& -4 \Omega_{\mu i} G_{i k} \Omega_{\mu l} G_{l r} \Omega_{\mu j} G_{j s} \Omega_{\mu k r s} \\
& -12 \Omega_{\mu i_{3}} G_{i_{3} j_{3}} \Omega_{\mu i_{2}} G_{i_{2} j_{2}} \Omega_{\mu j_{3} i_{1}} G_{i_{1} j_{1}} \Omega_{\mu j_{1} j_{2}} \\
& +12 \Omega_{\mu i_{1}} G_{i_{1} i_{2}} \Omega_{\mu i_{2} l} G_{l k} \Omega_{\mu j} G_{j i} \Omega_{\mu j_{1}} G_{j_{1} j_{2}} \Omega_{k i j_{2}} \\
& -3 \Omega_{\mu i_{1}} G_{i_{1} i_{2}} \Omega_{\mu i_{3}} G_{i_{3} i_{4}} \Omega_{i_{2} i_{4} l} G_{l k} \Omega_{\mu j} G_{j i} \Omega_{\mu j_{1}} G_{j_{1} j_{2}} \Omega_{k i j_{2}} \\
& +\Omega_{\mu u} G_{u k} \Omega_{\mu i} G_{i r} \Omega_{\mu l} G_{l s} \Omega_{\mu j} G_{j t} \Omega_{k r s t} .
\end{aligned}
$$

We have also used (D8) and (D13) to compute higher moments. A potential problem arises when the determinant of $\Omega^{(2)}$ in D7 is vanishing, which hampers the inverse of $\Omega^{(2)}$ and results in singularity for the propagator $G_{i j}$. Therefore, computations based on this analytical method need further investigations. 

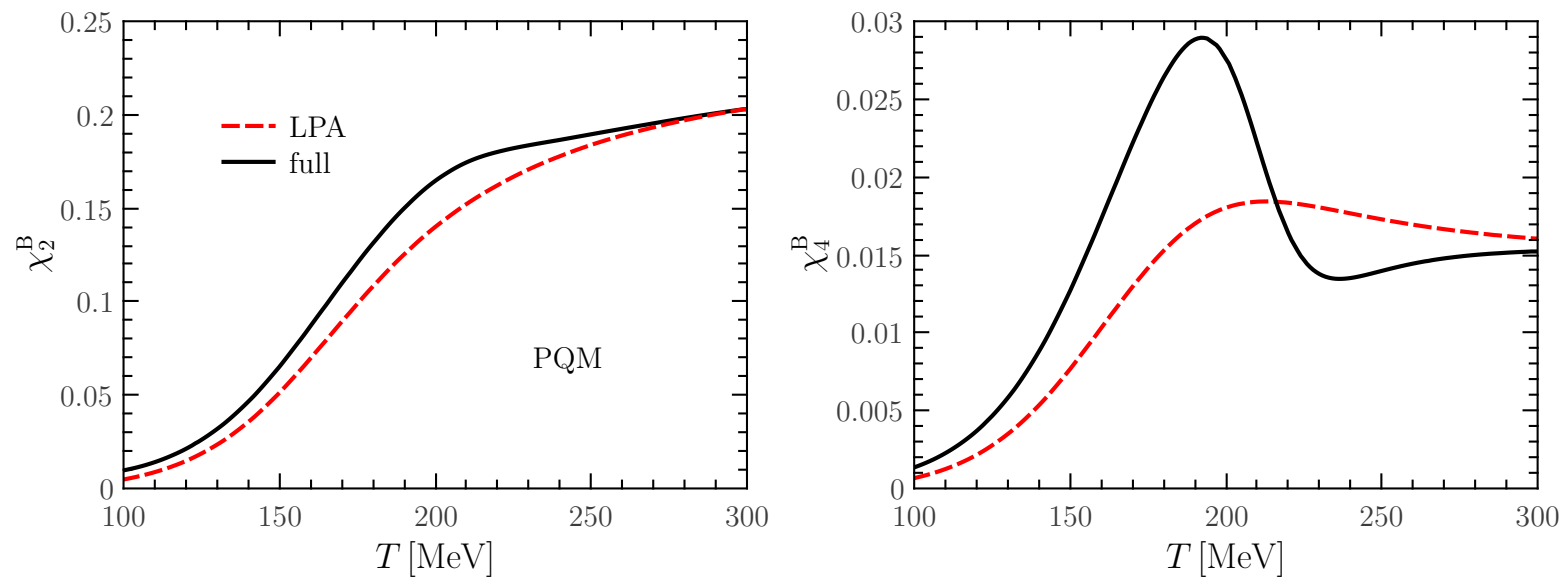

FIG. 18. Quadratic (left panel) and quartic (right panel) baryon number fluctuations as functions of $T$ calculated in the simplified PQM model, with the dependence of Polyakov loop on $T$ as input. Here we compare two different truncations: LPA and full.
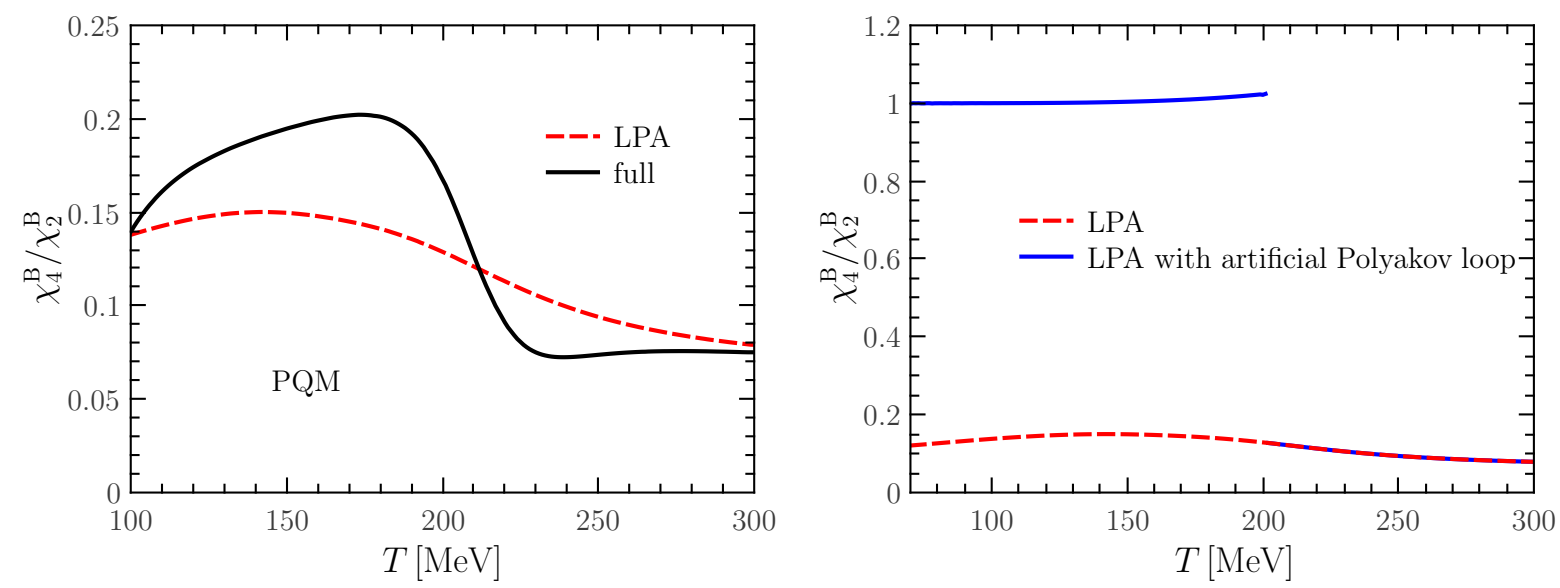

FIG. 19. Left panel: kurtosis of the baryon number distribution in the simplified PQM model. Right panel: the red-dashed curve is just the same one in the left panel, in comparison to the blue-solid line, which is obtained with the Polyakov loop set to be zero artificially below $T=200 \mathrm{MeV}$, while unchanged above this temperature. See text for more details.

\section{Appendix E: Kurtosis in a simplified PQM model}

The representation of the higher moments in Appendix D suggests the potential importance of a selfconsistent computation of all quantities, in particular that of the higher $L, \bar{L}$-moments of the glue potential. Here we check this hypothesis in the simplified PQM model: the $T$-dependent Polyakov loop expectation value is used as an input, and its dependence on the chemical potential is neglected. As external input we use the Polyakov loop expectation value as computed in 22 . Note that the LPA computation with a full effective meson potential there is very close to the present LPA computation within a Taylor expansion.

Fig. 18 shows the quadratic and quartic baryon number fluctuations in the simplified PQM model within LPA and full approximation. One observes that the fluctu- ations in the improved approximation are larger, and vary more rapidly with the change of temperature during the chiral crossover, similarily to the results in the self-consistent computation, see Fig. 13.

The kurtosis in the simplified PQM model, however, looks similar to that in the QM model: the missing selfconsistency of the glue background leads to a qualitative failure in the hadronic phase, see Fig. 19 in comparison to the self-consistent results in Fig. 14. Instead, the kurtosis in Fig. 19 is similar to that in the QM model, in agreement to an earlier mean field analysis in [37, see Fig. 5 and Fig. 7 there. If we enforce the hadronic nature with $L \bar{L}=0$ for $T \leq T_{c}$ and $L \bar{L}=1$ for $T \geq T_{c}$ the asymptotic low and high temperature behaviour is restored, see the right panel of Fig. 19. Note however, that this limit does not hold in the hadronic phase, instead we have (46) and (47). 
[1] D. J. Schwarz, Annalen Phys. 12, 220 (2003), arXiv:astro-ph/0303574 [astro-ph]

[2] J. Adams et al. (STAR Collaboration), Nucl.Phys. A757, 102 (2005), arXiv:nucl-ex/0501009 [nucl-ex].

[3] K. Adcox et al. (PHENIX Collaboration), Nucl.Phys. A757, 184 (2005), arXiv:nucl-ex/0410003 [nucl-ex]

[4] K. Aamodt et al. (ALICE Collaboration), Phys.Rev.Lett. 105, 252302 (2010), arXiv:1011.3914 [nucl-ex]

[5] M. Stephanov, PoS LAT2006, 024 (2006), arXiv:heplat/0701002 [hep-lat]

[6] S. Borsanyi, Z. Fodor, S. Katz, S. Krieg, C. Ratti, et al., Phys.Rev.Lett. 111, 062005 (2013), arXiv:1305.5161 [hep-lat]

[7] H.-T. Ding, Proceedings, 24th International Conference on Ultra-Relativistic Nucleus-Nucleus Collisions (Quark Matter 2014), Nucl. Phys. A931, 52 (2014), arXiv:1408.5236 [hep-lat]

[8] R. Bellwied, S. Borsanyi, Z. Fodor, S. D. Katz, A. Pasztor, C. Ratti, and K. K. Szabo, (2015), arXiv:1507.04627 [hep-lat]

[9] H. T. Ding, S. Mukherjee, H. Ohno, P. Petreczky, and H. P. Schadler, (2015), arXiv:1507.06637 [hep-lat]

[10] F. Karsch and K. Redlich, Phys. Lett. B695, 136 (2011), arXiv:1007.2581 [hep-ph]

[11] D. F. Litim and J. M. Pawlowski, , 168 (1998), arXiv:hep-th/9901063 [hep-th].

[12] J. Berges, N. Tetradis, and C. Wetterich, Phys. Rept. 363, 223 (2002) arXiv:hep-ph/0005122

[13] J. M. Pawlowski, Annals Phys. 322, 2831 (2007) arXiv:hep-th/0512261 [hep-th].

[14] H. Gies, Lect.Notes Phys. 852, 287 (2012), arXiv:hep$\mathrm{ph} / 0611146$ [hep-ph]

[15] B.-J. Schaefer and J. Wambach, Phys.Part.Nucl. 39, 1025 (2008), arXiv:hep-ph/0611191 [hep-ph].

[16] J. M. Pawlowski, AIP Conf.Proc. 1343, 75 (2011) arXiv:1012.5075 [hep-ph]

[17] J. Braun, J.Phys. G39, 033001 (2012), arXiv:1108.4449 [hep-ph]

[18] L. von Smekal, Nucl.Phys.Proc.Suppl. 228, 179 (2012), arXiv:1205.4205 [hep-ph]

[19] J. Braun, L. M. Haas, F. Marhauser, and J. M. Pawlowski, Phys.Rev.Lett. 106, 022002 (2011), arXiv:0908.0008 [hep-ph]

[20] J. Braun, Phys.Rev. D81, 016008 (2010) arXiv:0908.1543 [hep-ph]

[21] L. M. Haas, R. Stiele, J. Braun, J. M. Pawlowski, and J. Schaffner-Bielich, Phys.Rev. D87, 076004 (2013), arXiv:1302.1993 [hep-ph]

[22] T. K. Herbst, M. Mitter, J. M. Pawlowski, B.-J. Schaefer, and R. Stiele, Phys.Lett. B731, 248 (2014), arXiv:1308.3621 [hep-ph].

[23] J. M. Pawlowski and F. Rennecke, Phys. Rev. D 90, 076002 (2014), arXiv:1403.1179 [hep-ph]

[24] A. J. Helmboldt, J. M. Pawlowski, and N. Strodthoff, Phys.Rev. D91, 054010 (2015), arXiv:1409.8414 [hep$\mathrm{ph}]$

[25] M. Mitter, J. M. Pawlowski, and N. Strodthoff, Phys.Rev. D91, 054035 (2015), arXiv:1411.7978 [hep$\mathrm{ph}]$

[26] J. Braun, L. Fister, J. M. Pawlowski, and F. Rennecke, (2014), arXiv:1412.1045 [hep-ph].
[27] J. M. Pawlowski, Nucl.Phys. A931, 113 (2014).

[28] V. Skokov, B. Stokic, B. Friman, and K. Redlich, Phys.Rev. C82, 015206 (2010), arXiv:1004.2665 [hepph]

[29] V. Skokov, B. Friman, and K. Redlich, Phys.Rev. C83, 054904 (2011), arXiv:1008.4570 [hep-ph],

[30] B. Friman, F. Karsch, K. Redlich, and V. Skokov, Eur. Phys. J. C71, 1694 (2011), arXiv:1103.3511 [hep-ph]

[31] V. Skokov, B. Friman, and K. Redlich, Phys. Lett. B708, 179 (2012), arXiv:1108.3231 [hep-ph].

[32] V. Skokov, B. Friman, and K. Redlich, Phys. Rev. C88, 034911 (2013), arXiv:1205.4756 [hep-ph].

[33] K. Morita, V. Skokov, B. Friman, and K. Redlich, Eur.Phys.J. C74, 2706 (2014), arXiv:1211.4703 [hep-ph]

[34] K. Morita, B. Friman, K. Redlich, and V. Skokov, Phys.Rev. C88, 034903 (2013), arXiv:1301.2873 [hep$\mathrm{ph}]$,

[35] K. Morita, B. Friman, and K. Redlich, Phys. Lett. B741, 178 (2015), arXiv:1402.5982 [hep-ph].

[36] K. Morita and K. Redlich, PTEP 2015, 043 D03 (2015) arXiv:1409.8001 [hep-ph],

[37] W.-j. Fu, Y.-X. Liu, and Y.-L. Wu, Phys.Rev. D81, 014028 (2010), arXiv:0910.5783 [hep-ph].

[38] W.-j. Fu and Y.-l. Wu, Phys. Rev. D82, 074013 (2010) arXiv:1008.3684 [hep-ph]

[39] V. Skokov, B. Friman, E. Nakano, K. Redlich, and B.-J. Schaefer, Phys.Rev. D82, 034029 (2010) arXiv:1005.3166 [hep-ph]

[40] F. Karsch, B.-J. Schaefer, M. Wagner, and J. Wambach, Phys.Lett. B698, 256 (2011), arXiv:1009.5211 [hep-ph]

[41] B. J. Schaefer and M. Wagner, Phys. Rev. D85, 034027 (2012), arXiv:1111.6871 [hep-ph].

[42] M. Wagner, A. Walther, and B.-J. Schaefer, Comput.Phys.Commun. 181, 756 (2010), arXiv:0912.2208 [hep-ph]

[43] H. Gies and C. Wetterich, Phys.Rev. D65, 065001 (2002), arXiv:hep-th/0107221 [hep-th].

[44] H. Gies and C. Wetterich, Phys.Rev. D69, 025001 (2004), arXiv:hep-th/0209183 [hep-th],

[45] S. Floerchinger and C. Wetterich, Phys.Lett. B680, 371 (2009), arXiv:0905.0915 [hep-th],

[46] D. F. Litim, Phys.Lett. B486, 92 (2000), arXiv:hepth/0005245 [hep-th]

[47] D. F. Litim, Phys.Rev. D64, 105007 (2001), arXiv:hepth/0103195 [hep-th]

[48] G. Papp, B.-J. Schaefer, H. Pirner, and J. Wambach, Phys.Rev. D61, 096002 (2000), arXiv:hep-ph/9909246 [hep-ph].

[49] N. Mueller and J. M. Pawlowski, Phys. Rev. D91, 116010 (2015), arXiv:1502.08011 [hep-ph].

[50] K. Fukushima, Phys.Lett. B591, 277 (2004), arXiv:hepph/0310121 [hep-ph]

[51] C. Ratti, M. A. Thaler, and W. Weise, Phys.Rev. D73, 014019 (2006), arXiv:hep-ph/0506234 [hep-ph].

[52] W.-j. Fu, Z. Zhang, and Y.-X. Liu, Phys.Rev. D77, 014006 (2008), arXiv:0711.0154 [hep-ph]

[53] B.-J. Schaefer, J. M. Pawlowski, and J. Wambach, Phys.Rev. D76, 074023 (2007), arXiv:0704.3234 [hepph]

[54] C. S. Fischer, J. Luecker, and C. A. Welzbacher, (2014), arXiv:1405.4762 [hep-ph]. 
[55] P. M. Lo, B. Friman, O. Kaczmarek, K. Redlich, and C. Sasaki, Phys.Rev. D88, 014506 (2013), arXiv:1306.5094 [hep-lat]

[56] P. M. Lo, B. Friman, O. Kaczmarek, K. Redlich, and C. Sasaki, Phys.Rev. D88, 074502 (2013), arXiv:1307.5958 [hep-lat]

[57] J. Braun, H. Gies, and J. M. Pawlowski, Phys.Lett. B684, 262 (2010), arXiv:0708.2413 [hep-th]

[58] L. Fister and J. M. Pawlowski, Phys.Rev. D88, 045010 (2013), arXiv:1301.4163 [hep-ph].

[59] C. S. Fischer, L. Fister, J. Luecker, and J. M. Pawlowski, Phys.Lett. B732, 273 (2014) arXiv:1306.6022 [hep-ph]

[60] J. Greensite, Phys.Rev. D86, 114507 (2012) arXiv:1209.5697 [hep-lat]

[61] K. Langfeld and J. M. Pawlowski, (2013), arXiv:1307.0455 [hep-lat]

[62] D. Smith, A. Dumitru, R. Pisarski, and L. von Smekal, Phys.Rev. D88, 054020 (2013), arXiv:1307.6339 [heplat]

[63] D. Diakonov, V. Petrov, H.-P. Schadler, and C. Gattringer, JHEP 1311, 207 (2013), arXiv:1308.2328 [heplat]
[64] J. Greensite and K. Langfeld, Phys. Rev. D90, 014507 (2014), arXiv:1403.5844 [hep-lat].

[65] W.-j. Fu, T. K. Herbst, J. M. Pawlowski, F. Rennecke, and B.-J. Schaefer, in progress (2015).

[66] S. Borsanyi, G. Endrodi, Z. Fodor, A. Jakovac, S. D. Katz, et al., JHEP 1011, 077 (2010), arXiv:1007.2580 [hep-lat].

[67] S. Borsanyi et al. (Wuppertal-Budapest Collaboration), JHEP 1009, 073 (2010), arXiv:1005.3508 [hep-lat]

[68] L. Adamczyk et al. (STAR), Phys.Rev.Lett. 112, 032302 (2014), arXiv:1309.5681 [nucl-ex].

69] FQCD Collaboration, J. Braun, A.K. Cyrol, L. Fister, W.-J. Fu, T.K. Herbst, M. Mitter, J.M. Pawlowski, F. Rennecke, and N. Strodthoff.

[70] N. Christiansen, B. Knorr, J. M. Pawlowski, and A. Rodigast, (2014), arXiv:1403.1232 [hep-th].

[71] N. Christiansen, B. Knorr, J. Meibohm, J. M. Pawlowski, and M. Reichert, (2015), arXiv:1506.07016 [hep-th].

[72] L. Fister and J. M. Pawlowski, (2015), arXiv:1504.05166 [hep-ph]. 